\title{
Colombia: Términos de intercambio y fluctuaciones de la producción
}

\author{
Gonzalo Hernández
}

RESUMEN

En este artículo se explora la incidencia de los términos de intercambio en las fluctuaciones de la producción en Colombia, un país en desarrollo cuyos ingresos por exportaciones proceden mayoritariamente (en un 62\%) de solo cuatro productos básicos: el petróleo (42\%), el carbón (14\%), el café (5\%) y el níquel (1\%). Esta investigación fue motivada por el papel especial que desempeñan las fluctuaciones de corto plazo en las economías en desarrollo, por el hecho de que los términos de intercambio de Colombia sean procíclicos y por la discusión acerca de las políticas económicas orientadas a esterilizar los efectos de los precios de los productos básicos. Tras un análisis de series cronológicas del período 1994-2011, se hallaron pruebas sólidas de que alrededor de un tercio del crecimiento trimestral de la economía colombiana es atribuible a variaciones de los términos de intercambio.

PALABRAS CLAVE

CLASIFICACIÓN JEL

AUTOR
Comercio internacional, relación de intercambio, crecimiento económico, productividad, análisis de series cronológicas, Colombia

F41, F44, O54

Gonzalo Hernández es profesor del Departamento de Economía de la Pontificia Universidad Javeriana de Bogotá y $\mathrm{PhD}$ (c) en el Departamento de Economía de la Universidad de Massachusetts, Amherst (Estados Unidos).gonzalo@econs.umass.edu 


\section{I}

\section{Introducción}

El papel de las fluctuaciones de corto plazo de la producción en las economías en desarrollo es especialmente significativo. Los países en desarrollo suelen estar más expuestos que las economías desarrolladas a los efectos de los altibajos macroeconómicos. Además, las consecuencias en materia de bienestar pueden ser asimétricas, en función del grado de desarrollo. Algunos ejemplos de posibles efectos desiguales serían, en primer lugar, que las economías de los países en desarrollo carecen de redes de seguridad social adecuadas para mitigar el impacto de las fases negativas en los sectores más pobres de la población; segundo, que en los países en desarrollo la pobreza y el desempleo restringen la capacidad de las personas para ajustar su consumo cuando se producen perturbaciones temporales ${ }^{1}$; y en tercer lugar, que la mayor variabilidad de la base fiscal puede limitar el margen del sector público para implementar los proyectos de largo plazo necesarios con el fin de eliminar los obstáculos al desarrollo de estas economías, además de reducir la capacidad de respuesta de la política fiscal en el corto plazo.

Este estudio se centra en los términos de intercambio para explicar esas fluctuaciones de la producción. La decisión de adoptar este enfoque tiene sus motivaciones en la bibliografía sobre la macroeconomía del desarrollo basada en un marco económico pequeño y abierto ${ }^{2}$. En particular, según el modelo de la economía dependiente (con su variante de tres tipos de bienes: exportables, importables y no comerciables) las pequeñas economías enfrentan una demanda global de sus productos y una oferta de productos importados infinitamente elásticas ${ }^{3}$,

$\square$ El autor desea manifestar su agradecimiento a Arslan Razmi, Gerald Epstein, Christian Rojas, Leila Davis, Martín Rapetti y a un árbitro anónimo por sus útiles comentarios a borradores previos. Las opiniones expresadas en este artículo son exclusivamente del autor.

${ }^{1}$ En la teoría económica, bajo los supuestos de aversión al riesgo y mercados financieros incompletos, normalmente se asume que una mayor volatilidad en el consumo reduce la utilidad de los individuos. 2 Véase, por ejemplo, Agénor y Montiel (2008).

3 Para los efectos de este estudio, la economía dependiente con tres tipos de bienes parece ser un marco más conveniente que el modelo Mundell-Fleming, en que los términos de intercambio son endógenos cuando son variables. La endogeneidad del modelo Mundell-Fleming se debe a la existencia de cierto poder del mercado productor en el bien exportable. El precio del bien exportable puede verse alterado por condiciones internas (es decir, la demanda interna) incluso si la economía no influye en los precios de los productos importables. Del mismo modo, el modelo de economía dependiente con dos tipos lo que significa que los precios de las exportaciones y las importaciones se determinan en los mercados internacionales en los que la economía nacional no tiene influencia. El marco predice que las perturbaciones externas a los términos de intercambio pueden ser una importante fuente de fluctuaciones de la producción en la economía nacional. La mejora de los términos de intercambio — debida, por ejemplo, a un alza de los precios de los productos básicos-opera como un incentivo para aumentar la producción en los sectores que resultan beneficiados con un precio más alto. Sin embargo, la perturbación puede provocar también una apreciación del tipo de cambio real que repercuta en un incremento de los salarios reales en los sectores que compiten con productos importables. Por lo tanto, el aumento inicial de la producción agregada podría quedar contrarrestado por la pérdida de competitividad en los sectores que compiten con productos importables (enfermedad holandesa). El mismo mecanismo se puede aplicar fácilmente a otros productos exportables. Sin embargo, el resultado neto depende teóricamente de supuestos fundamentales sobre los mercados de trabajo y el grado de flexibilidad de los precios (la situación del mercado). La suposición más común es que la compensación en el sector no exportador se realiza por las variaciones de precios y no a través de un ajuste de la producción. Además, que estos efectos se manifiesten en el corto plazo no solo dependerá del tipo de ajuste del mercado, sino también de su rapidez, la reacción de las autoridades de la política económica a los cambios de este precio relativo, el grado de apertura, el grado de especialización en bienes exportables y el régimen cambiario, entre otros elementos.

Algunos hechos justifican la selección de Colombia como caso de estudio. En primer lugar, la estructura de las exportaciones colombianas recientes parece apoyar el papel de los términos de comercio exógenos como en el modelo de tres tipos de bienes. Los datos del año 2010 muestran que las exportaciones colombianas

de bienes (comercializados y no comercializados) tiene su propia limitación: tanto los bienes exportables como los importables quedan agregados en un bien compuesto (el bien comercializado). Por lo tanto, la variabilidad de los términos de intercambio no está definida y no puede ser el origen de las fluctuaciones macroeconómicas. Véanse, por ejemplo, Greenwood (1984) y Buiter (1988) para conocer otras especificaciones de modelos de economía dependiente. 
se concentran principalmente (en un 62\%) en cuatro productos básicos respecto de los cuales el poder de mercado de Colombia no es significativo: petróleo y derivados $(42 \%)$, carbón $(14 \%)$, café $(5 \%)$ y níquel (1\%). En segundo lugar, los datos trimestrales a lo largo del período 1994-2011 indican una correlación positiva entre las variaciones trimestrales de los términos de intercambio y el crecimiento trimestral del producto interno bruto (PIB) es igual a 0,35. Esta magnitud es importante en relación con otros estudios sobre las economías en desarrollo (véase la sección II). Por último, en el período 1994-2011 se registra una alta variabilidad tanto en los términos de intercambio como en el PIB. Esta variabilidad es útil para comprobar la validez de los resultados del análisis de series cronológicas.

Aunque este estudio se limita a los resultados agregados, hay otros canales que pueden ilustrar la relevancia de los términos de intercambio en el desempeño económico de Colombia en el corto plazo. Si se toma nuevamente el alza de precios de los productos básicos como ejemplo, cuando aumente el precio de uno de estos productos se generarán beneficios adicionales para las empresas involucradas directa o indirectamente en esa producción. Por lo tanto, la turbulencia favorece en este caso a la expansión del consumo y la producción en otros sectores. Además, un mayor nivel de riqueza facilita a los inversores el acceso al crédito, a consecuencia de una mayor disponibilidad de divisas que reduce las restricciones monetarias. Por consiguiente, el proceso impulsa al crédito, la inversión y el consumo, y también genera utilidades para el sistema financiero, que en la actualidad representa alrededor de un $18 \%$ del valor agregado total de Colombia. Este mecanismo es claramente plausible, ya que en el país se ha producido una gran acumulación de reservas internacionales y, a pesar de la política de fijación de metas de inflación del banco central, se han realizado algunas intervenciones para contener la apreciación del tipo de cambio.

Otra de las explicaciones de esta tendencia procíclica de los términos de intercambio en Colombia se puede encontrar en el sector público. Alrededor del $60 \%$ del volumen total de petróleo exportado corresponde a la Empresa Colombiana de Petróleo (ECOPETROL). Algunos de los ingresos obtenidos por esta empresa forman parte de los ingresos del sector público no financiero. Además, es razonable esperar que aumentando los beneficios de las empresas y estimulando la economía, con el alza de los precios de los productos básicos también se amplíen los ingresos fiscales. Eso no necesariamente se traduciría en un superávit fiscal. Por ejemplo, Kaminsky (2010) encuentra evidencias de una política fiscal procíclica en países de ingresos medianos cuando se producen turbulencias en los términos de intercambio 4 .

En cuanto al comercio internacional, después de los Estados Unidos de América y la Unión Europea, Venezuela (República Bolivariana de) y el Ecuador son los mercados más importantes para las exportaciones colombianas. Estos dos países son exportadores de petróleo y compradores netos de manufacturas colombianas. Esto significa que un alza de precios de los productos básicos que aumente los ingresos de estos socios comerciales también puede provocar un incremento de la demanda de productos colombianos. Sin embargo, los datos preliminares indican que la cuenta corriente de la balanza de pagos del país no se correlaciona positivamente con los términos de intercambio ${ }^{5}$.

En este estudio se intenta resolver la relación teóricamente ambigua que existe entre los términos de intercambio y la producción. En concreto, se presenta un análisis de series cronológicas en que se examina la relación entre el crecimiento del PIB trimestral y las variaciones trimestrales de los términos de intercambio. Con esa finalidad, se ha creado un índice de precios para los cuatro principales productos básicos exportables y se ha utilizado una metodología econométrica simple (BoxJenkins), en línea con la exogeneidad de los términos de intercambio de Colombia, la no cointegración entre el PIB y los términos de intercambio y la estabilidad de las variables clave. En el trabajo se presentan distintas pruebas de robustez, empezando por la inclusión de las variables de control significativas cuya ausencia puede provocar un sesgo en la estimación de los términos de intercambio. Por ejemplo, los tipos de cambio real y nominal son dos variables de control significativas, debido a que un efecto negativo de las variaciones de los términos de intercambio en las fluctuaciones de la producción en el corto plazo podría estar vinculado a un fenómeno de enfermedad holandesa. Sin embargo, no está claro a priori que las perturbaciones positivas de los términos de intercambio provoquen una apreciación o que con esta se vaya a reducir el producto agregado de modo inequívoco. En primer lugar, la producción de bienes que no se comercializan podría aumentar debido a dichas perturbaciones, mientras que la expansión de los ingresos reales se ajusta mediante un cambio en

\footnotetext{
4 Véanse, por ejemplo, Tornell y Lane (1999) y Frankel (2010) para conocer aspectos institucionales que explican el gasto público procíclico en las economías en desarrollo. Con relación a América Latina, véase, por ejemplo, Medina (2010).

5 Véanse en Obstfeld (1982); Svensson y Razin (1983) y Kent y Cashin (2003) discusiones acerca de los efectos de los términos de intercambio en la cuenta corriente de la balanza de pagos.
} 
la producción y no en los precios. En segundo lugar, una eventual apreciación del tipo de cambio nominal ante una mayor oferta de divisas podría tener efectos expansivos en la producción, así como una devaluación nominal puede tener efectos contractivos. En un modelo teórico seminal, Krugman y Taylor (1978) describen esta posibilidad $^{6}$. En uno de los mecanismos presentados

\footnotetext{
${ }^{6}$ Véase en Lizondo y Montiel (1989) una descripción detallada de la teoría de los efectos contractivos de la devaluación aplicada a los países en desarrollo. Razmi (2007) amplía el marco teórico de Krugman y Taylor (1978), al incluir el papel de las empresas transnacionales y el tipo de socios comerciales para las exportaciones (economía en desarrollo o industrializada). A partir de ahí sugiere que la probabilidad de que la devaluación tenga efectos contractivos en el corto plazo puede ser mayor en las economías en desarrollo. Por el contrario, Reinhart y Reinhart (1991) observan que una devaluación es expansiva en el corto plazo en Colombia mediante un modelo basado en simulaciones con una estructura neokeynesiana.
}

por estos autores se establece, en consonancia con la tradición kaleckiana, que con una apreciación se pueden redistribuir hacia los salarios los ingresos por beneficios y arriendos. La reducción del precio de los insumos importados se traduce automáticamente en un descenso del precio de los productos del país, con lo que se incrementan los salarios reales. Dado que la propensión marginal a consumir de los trabajadores es mayor que la de los capitalistas, la redistribución de los salarios en los beneficios aumenta la demanda agregada y la producción nacional.

Por lo tanto, luego de reseñar la bibliografía relacionada en la sección II, la estrategia empírica presentada en la sección III es esencial para evaluar el efecto directo de las variaciones de los términos de intercambio después de considerar los posibles efectos indirectos mediante otras variables. Las observaciones finales se presentan en la sección IV.

\section{II}

\section{Bibliografía relacionada}

Las pruebas empíricas de los efectos de los términos de intercambio en las fluctuaciones de la producción en las economías en desarrollo se pueden clasificar en tres grupos: i) estudios en que se describe una correlación entre ciclos económicos y ciclos de los términos de intercambio como un hecho estilizado; ii) modelos basados en simulaciones, y iii) modelos de autorregresión vectorial (VAR, por sus siglas en inglés).

Agénor, McDermott y Prasad (2000) detectan, por ejemplo, una pronunciada correlación positiva en los casos de Colombia, la República de Corea y México entre los componentes cíclicos de la producción industrial y los términos de intercambio (empleando tanto la metodología de Hodrick Prescott como la de paso de banda con datos trimestrales). También en este grupo de artículos, Parra (2008), con datos trimestrales desde 1994 hasta 2007, informa de una correlación igual a 0,24 para Colombia; y Mahadeva y Gómez (2009), da cuenta de una correlación positiva entre los términos de intercambio y el PIB real per cápita de Colombia igual a 0,32 (al utilizar datos anuales para 1970-2007) ${ }^{7}$. Sin embargo, este tipo de

\footnotetext{
7 Véase en Rand y Tarp (2002) una descripción de hechos estilizados de los ciclos económicos en los países en desarrollo.
}

hecho estilizado resulta más convincente cuando se utiliza para la calibración de modelos basados en simulaciones o para la especificación de un modelo econométrico.

Por ejemplo, Mendoza (1995), en un trabajo seminal que se inscribe en la segunda categoría, no solo informa de una correlación positiva entre los términos de intercambio y el PIB, sino que afirma además que su modelo intertemporal predice que las perturbaciones de los términos de intercambio pueden suponer entre el $37 \%$ y el $56 \%$ de la variabilidad del PIB en los países en desarrollo. Por supuesto, este resultado depende de la configuración específica de su modelo de tres tipos de productos (bienes exportables, importables y no comercializados). En ese marco, el efecto dominante que explica la incidencia en el corto plazo de los términos de intercambio en la producción consiste, básicamente, en que las mejoras en la relación de intercambio inducen un aumento de la rentabilidad marginal del sector de bienes exportables, lo que estimula el auge de las inversiones en este sector. Las inversiones corresponden a una reasignación nacional e internacional de capital, en que el sector de los bienes importables es la única fuente de capital nacional (no el sector de bienes no comercializados). Por otra parte, la oferta de mano de obra es inelástica en las industrias del sector de bienes comercializados, mientras que en el de 
bienes no comercializados se supone que la respuesta a la oferta de mano de obra no es significativa. Después del efecto en el corto plazo, los mecanismos de ajuste empiezan a operar para conducir la economía a un equilibrio en el largo plazo, que por definición es igual al equilibrio inicial. El ajuste del tipo de cambio real hacia su equilibrio en el largo plazo reduce el diferencial de las tasas de interés en el corto plazo y, por lo tanto, se retira el capital extranjero que ingresó en la economía del país durante el auge de la inversión. Como era de esperar, se debilita así la expansión del PIB que se había iniciado.

A pesar de que el marco de Mendoza (1995) presenta un escenario plausible para la correlación positiva entre los términos de intercambio y el PIB en el corto plazo, los distintos supuestos teóricos podrían ofrecer otras versiones. De hecho, desde un punto de vista empírico, algunos países presentan una correlación negativa a partir de su propia muestra: Argelia $(-0,234)$, Egipto $(-0,455)$, Filipinas $(-0,285)$, República Democrática del Congo $(-0,107)$ y Túnez $(-0,309)$. Sin embargo, en su artículo estos casos no quedan cubiertos por el modelo de equilibrio general.

Kose y Riezman (1999) y Kose (2002) ofrecen otros ejemplos de cómo la evidencia empírica puede estar condicionada por la configuración teórica particular. Kose y Riezman (1999), que desarrollan un modelo de equilibrio general para una pequeña economía africana abierta con dos sectores (bienes primarios exportables y bienes no comercializados), llegan a la conclusión de que las perturbaciones de los precios mundiales pueden explicar alrededor del $45 \%$ de las fluctuaciones de la producción, sobre todo porque tanto en el sector de bienes primarios como en los de bienes no comercializados se utilizan bienes de capital importados como factores de producción. Por lo tanto, una disminución de los precios internacionales de las importaciones conduce a una expansión de la producción agregada. Por otra parte, Kose (2002) considera que las perturbaciones de los precios de los bienes de capital y de bienes intermedios pueden representar hasta un $87,6 \%$ de la variabilidad de la producción. La mayor magnitud del efecto en este caso obedece a que el autor se centra en los precios de los principales productos de importación y exportación (que son más sensibles que los términos de intercambio a las perturbaciones de la productividad), así como en el papel de los insumos intermedios en el sector no comercializado, que - de acuerdo con su modelo de pequeña economía abierta - no se ve sometido a ningún límite en cuanto a la oferta de capital.

En el tercer grupo de estudios se han utilizado las técnicas del modelo VAR para examinar los efectos de los términos de intercambio en las fluctuaciones de la producción en los países en desarrollo; por ejemplo, en los estudios de Hoffmaister, Roldós y Wickham (1998); Hoffmaister y Roldós (2001); Ahmed (2003); Broda (2004); Izquierdo, Romero y Talvi (2007), y Raddatz (2007). Normalmente, estos análisis se basan en modelos teóricos de largo plazo cuyas formas reducidas se convierten en modelos VAR estructurales específicos. Por ejemplo, Hoffmaister, Roldós y Wickham (1998) señalan que las fluctuaciones de los términos de intercambio operan a través de los precios de los insumos intermedios, suponiendo que un cambio positivo en este precio se comporta como un avance tecnológico negativo. De esta manera, las perturbaciones positivas de los términos de intercambio son choques de oferta positivos que relajan la restricción de los insumos intermedios.

Independientemente de los aspectos específicos de la técnica, la mayoría de los autores sugieren un efecto positivo de los términos de intercambio en las fluctuaciones de la producción en los países en desarrollo. Sin embargo, en una parte de la bibliografía documentada se cuestiona el papel de los precios internacionales. Por ejemplo, Broda (2004) afirma que los datos empíricos de que dispone contradicen los de Mendoza (1995). Broda, que analizó una muestra de 75 países en desarrollo que incluía datos anuales de 1973 a 1996, observa que la contribución de las perturbaciones de los términos de intercambio representa menos del $10 \%$ de volatilidad del PIB real en los países con regímenes cambiarios flexibles. Asimismo, Ahmed (2003), que estudió las fluctuaciones económicas de seis países latinoamericanos: Argentina, Brasil, Chile, Colombia, México y Venezuela (República Bolivariana de), concluye que las perturbaciones de los términos de intercambio pueden representar (aunque sea significativo en términos estadísticos) menos del $8 \%$ de las fluctuaciones de la producción nacional.

Los términos de intercambio también se han utilizado como variable de control a la hora de explicar la relación entre las fluctuaciones de corto plazo del PIB y otras variables en los países de América Latina, por ejemplo, en Barro (1979) y Edwards (1983 y 1986). En consonancia con los estudios descritos anteriormente, el efecto suele ser positivo. Sin embargo, Edwards (1983) observa que la estimación del efecto de los términos de intercambio en la producción solo es importante para Chile y México, no para el Brasil, Colombia ni el Perú. Además, Edwards (1986), que comprueba si una devaluación del tipo de cambio nominal puede provocar efectos contractivos en el corto plazo, concluye que el efecto de la relación de intercambio en la producción real de los países en desarrollo es insignificante. Según el autor, en ningún 
estudio anterior se determina la medida en que se pueden atribuir a los términos de intercambio las fluctuaciones de la producción en el contexto colombiano reciente. Las características específicas de Colombia y su estatus de economía en desarrollo posibilitan el uso de una sencilla, pero potente herramienta econométrica para llevar a cabo esa cuantificación y comprobar su robustez. Dado que en la bibliografía constan varios casos de efectos positivos, negativos y nulos de los términos de intercambio, en el caso colombiano el examen es fundamentalmente empírico.

\section{III}

\section{Estrategia empírica}

Esta sección tiene por objeto ofrecer un modelo parsimonioso de Colombia en el período 1994-2011 para describir las fluctuaciones de su producción, estimar el efecto parcial de los términos de intercambio en las variaciones del PIB y probar la significación de esa estimación a partir de datos trimestrales.

Este período de análisis se seleccionó por varias razones. En primer lugar, los datos están disponibles sin cambios metodológicos sustanciales en las cuentas nacionales ni en la balanza de $\operatorname{pagos}^{8}$. En segundo lugar,

8 La información se obtuvo directamente del Departamento Administrativo Nacional de Estadística (DANE); entre los datos de las Estadísticas Financieras Internacionales no figuran datos trimestrales del PIB de Colombia hasta 1994. en el análisis se excluye a uno de los puntos de ruptura estructural más relevantes en la política económica colombiana: la liberalización del comercio a principios de los años noventa. En tercer lugar, el período incluye lo siguiente: el auge de los precios de los productos básicos que se inició en 2003, la posterior desaceleración a finales de 2008 (que afectó a los términos de intercambio de Colombia) y una recuperación a partir de 2009. Este período comprende, asimismo, la mayor recesión conocida en la historia colombiana (año 1999) y un período de alto crecimiento (2003-2007) (véase el gráfico 1).

En lo que se refiere al procedimiento estadístico, en este artículo se sigue la técnica de Box-Jenkins para un modelo univariante. El tipo de modelo cuya estimación se realiza se conoce generalmente en la literatura como

GRÁFICO 1

PIB real y términos de intercambio

(Escala logarítmica)
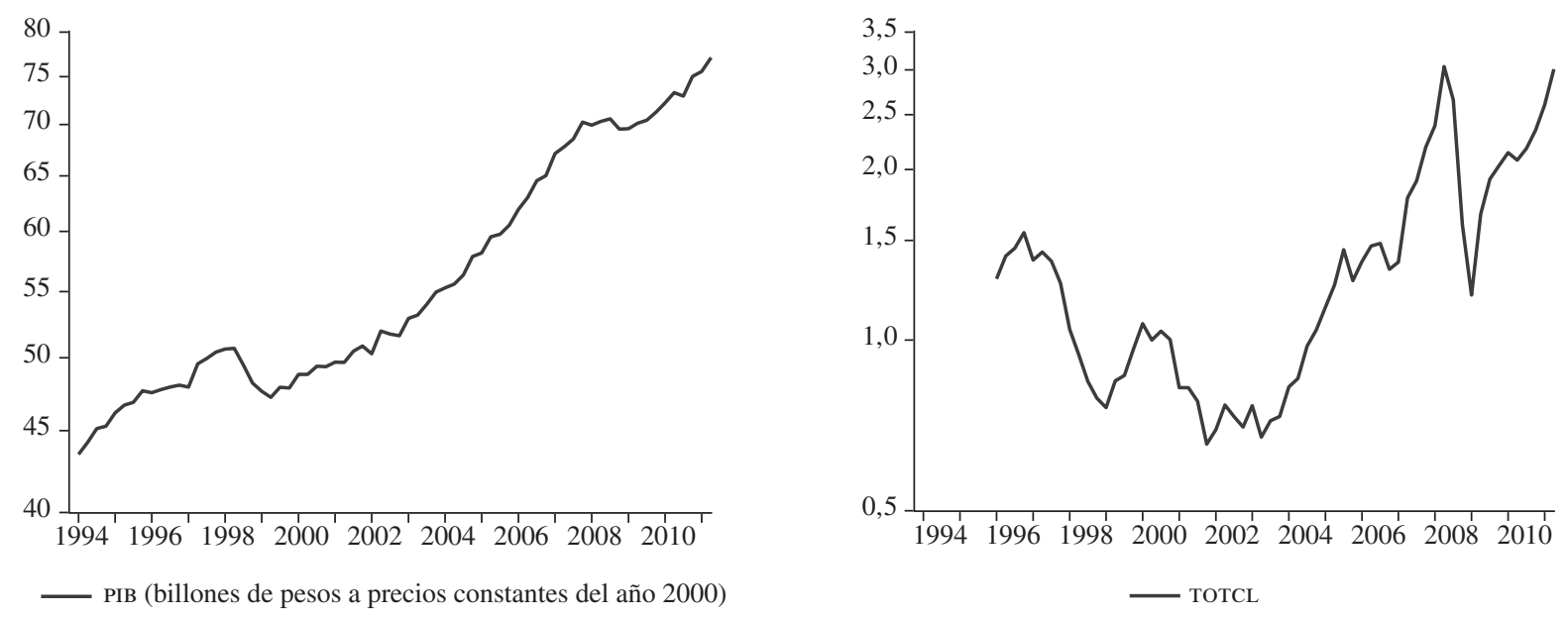

Fuente: Departamento Administrativo Nacional de Estadísticas (DANE), Banco de la República de Colombia (BRC) y cálculos del autor.

PIB: producto interno bruto.

тотCL: términos de intercambio de los cuatro productos básicos de exportación (petróleo, carbón, café y níquel). 
modelo autorregresivo de promedio móvil con variables exógenas (ARMAX), y es para series estacionarias con tres componentes: i) la parte autorregresiva (AR); ii) el promedio móvil (MA), y iii) el conjunto de las restantes variables explicativas (x). El modelo general es el siguiente:

$y_{t}=\alpha+\sum_{p=1}^{n} \lambda_{p} y_{t-p}+\sum_{q=1}^{n} \theta_{q} \mu_{t-q}+\sum_{m=0}^{n} \gamma_{i, m} X_{i, t-m}+\mu_{t}$

donde $y$ representa la variable dependiente (una serie estacionaria del PIB en este artículo), $t$ es un índice de tiempo, $\mu$ es el término de error, $X$ es el conjunto de variables explicativas (estacionarias y que incluye los términos de intercambio) y $\alpha, \lambda, \theta, \gamma$ son los parámetros cuya estimación se debe realizar.

Existen varias razones que justifican la especificación en la ecuación (1), teniendo en cuenta que el PIB y los términos de intercambio no están cointegrados (véase el cuadro A1 del anexo) ${ }^{9}$. En primer lugar, las series estacionarias reducen las posibilidades de que surjan correlaciones espurias debido a tendencias similares entre la variable dependiente y una variable explicativa. Segundo, la descomposición de Wold muestra que todo proceso estacionario se puede abordar mediante la combinación de los modelos autorregresivo y de promedio móvil. En tercer lugar, ambos componentes combinados contribuyen a la parsimonia del modelo, una vez que se ha tenido en cuenta la autocorrelación de los errores que afectarían a las pruebas de significación. Por último, la utilización de un modelo autorregresivo ARMA permite controlar cualquier posible persistencia de las fluctuaciones de la producción ${ }^{10}$. Además del efecto contemporáneo, esta especificación permite estimar el efecto total de los términos de intercambio en el PIB a lo largo del tiempo.

Además de la especificación del modelo ARMA y los términos de intercambio, deben considerarse las variables de control en el conjunto de variables explicativas, sobre todo porque su omisión puede dar lugar a un sesgo de estimación del efecto de los términos de intercambio. Por parte de la demanda agregada ${ }^{11}$, las pruebas de robustez incluyen dos grupos de variables

9 Véase, por ejemplo, Montenegro (2002).

10 Véanse, por ejemplo, Nelson y Plosser (1982); Campbell y Mankiw (1987), y Blanchard y Quah (1989) para obtener más información sobre la persistencia de las fluctuaciones de la producción.

11 Véase Shapiro y Watson (1989), en cuyo trabajo la fuente de las fluctuaciones de la producción se divide entre componentes de oferta y demanda. monetarias: las tasas de interés activas y los tipos de cambio. Las tasas de interés pueden ser importantes en la determinación del componente de la inversión, lo que explica en gran medida la variabilidad del PIB, y también pueden ser sensibles a las variaciones de los términos de intercambio a través de la disminución de las limitaciones de la balanza de pagos. Por otra parte, con la inclusión de los tipos de cambio nominales y reales no solo es posible probar la robustez de los efectos de los términos de intercambio, sino también examinar si el efecto de corto plazo de una depreciación (o de una apreciación) del tipo de cambio es contractivo (o expansivo). Por último, el crecimiento trimestral del PIB de los Estados Unidos de América (GDPUs) y las corrientes financieras netas (entradas menos salidas) en la balanza de pagos de Colombia (flujos financieros netos (NFF, por sus siglas en inglés)) también se tratarán como variables de control. Ambas variables se podrían también correlacionar simultáneamente con los términos de intercambio y el PIB colombiano.

La especificación deja de lado los shocks tecnológicos, que son un elemento importante en la literatura sobre ciclos económicos reales ${ }^{12}$. Esta decisión se justifica bajo tres aspectos. En primer lugar, dada la volatilidad de los datos trimestrales, los shocks tecnológicos relacionados con los términos de intercambio, y que pueden explicar las variaciones del PIB trimestre a trimestre, son poco probables (aunque se consideren algunos amplificadores). Segundo, los indicadores representativos del cambio técnico, como la productividad total de los factores, no suelen fiables, especialmente en los países en desarrollo. En tercer lugar, a pesar de que una identificación clara resulta imposible, con la especificación del modelo ARMA ya se está controlando la nueva información (las innovaciones) a través del término de promedio móvil, incluidas las perturbaciones no observables que afectan a la producción ${ }^{13}$.

12 Véase en Mankiw (1989) una crítica de la teoría del ciclo económico real y en Holland y Scott (1998) una defensa empírica de que el cambio técnico explica el ciclo económico en el Reino Unido de Gran Bretaña e Irlanda del Norte.

${ }^{13}$ El fenómeno de El Niño, otro shock de la oferta no correlacionado con los términos de intercambio, pero que podría resultar útil para comprender la naturaleza del ciclo económico colombiano, se examinó en un análisis anterior no señalado en este documento. Con el índice multivariado de El Niño/Oscilación Austral se utilizaron diferentes definiciones alternativas para crear una variable ficticia en función de si el trimestre coincidía o no con la fase cálida, si coincidía o no con una fase cálida cuyo índice era una desviación típica superior al promedio, o si el valor absoluto del índice era relativamente alto en comparación con el promedio. En el período analizado no se halló ninguna relación clara entre El Niño y el PIB. 
En el estudio no se tienen en cuenta las expectativas de los agentes económicos ni la gestión de esas expectativas como instrumento de política económica, por ejemplo, por medio de la política monetaria. Se trata de una limitación del estudio, ante la dificultad de encontrar un sustituto adecuado para esa variable.

$\mathrm{Si}$ bien en una investigación ulterior cabe la posibilidad de explorar si determinadas variables pueden mejorar la adecuación del modelo, el objetivo principal del estudio no es obtener un modelo para pronósticos, sino evaluar el papel de los términos de intercambio.

\section{Variables y descripción de los datos}

\section{- Producto interno bruto (PIB)}

Los datos trimestrales del PIB real, con ajuste estacional, se obtuvieron del DANE (véase el cuadro A2). La variable dependiente es la primera diferencia del logaritmo del PIB (DLGDP) de Colombia (aproximadamente el crecimiento trimestral del PIB) (véase el gráfico 2). Esta transformación es necesaria por dos razones: define la variable en términos de fluctuaciones de la producción y cumple el requisito de estacionariedad de la técnica Box-Jenkins. Según diferentes pruebas, se verifica una débil estacionariedad de DLGDP al rechazarse la hipótesis nula de que la serie tiene una raíz unitaria (véase el cuadro A3).

Como definición alternativa de las fluctuaciones de la producción, se realizó la estimación del componente cíclico del PIB (GDPCYCLE) mediante el filtro de HodrickPrescott. Esta serie también es estacionaria.

\section{—Términos de intercambio y precios relacionados}

En este artículo se utilizan dos definiciones de los términos de intercambio. La primera se basaba en información estadística de la balanza de pagos y del índice de precios al por mayor de las importaciones facilitada por el banco central de Colombia. Esta definición, denominada TOTCL, corresponde a la relación $(P X C L / P I)$, en que el denominador es el índice de precios al por mayor de las importaciones y el numerador es un índice de Laspeyres de la canasta de los principales

GRÁFICO 2

Primera diferencia del logaritmo del PIB

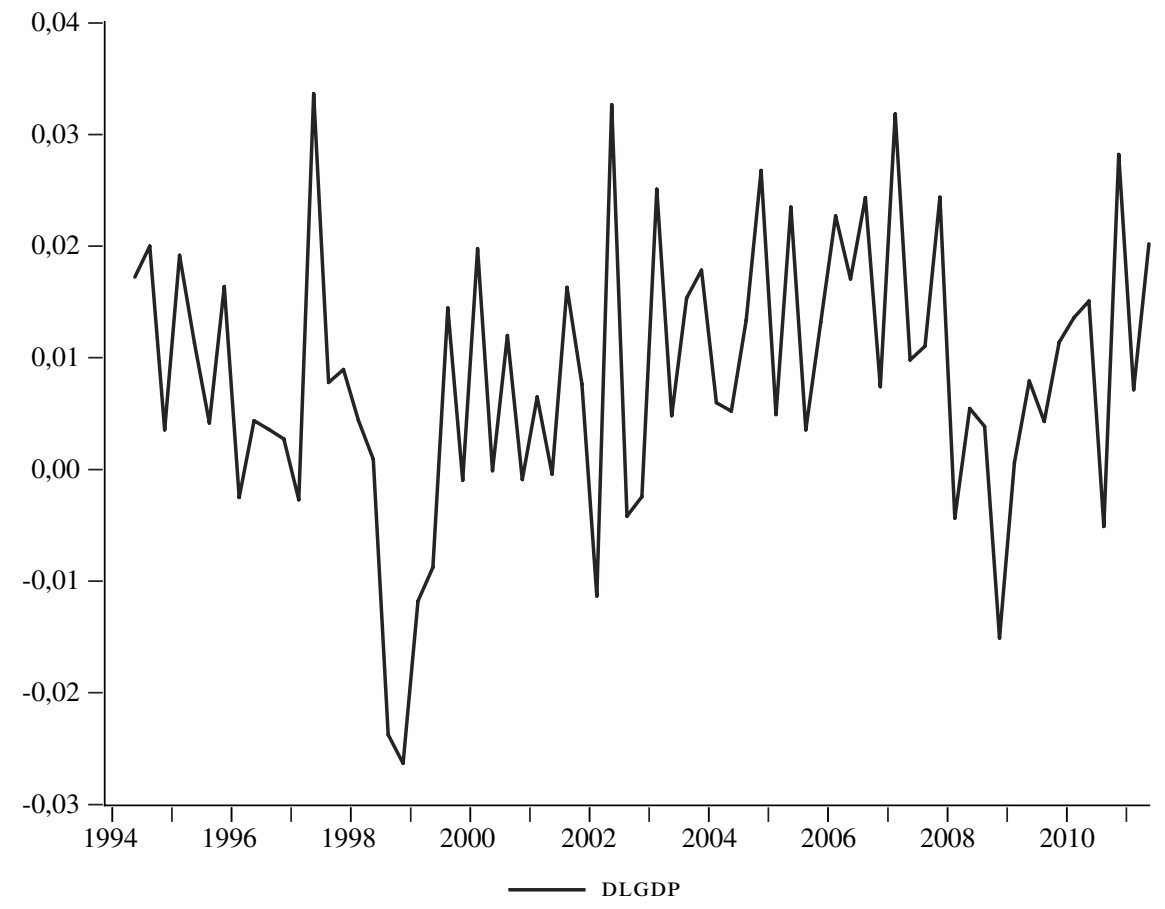

Fuente: Departamento Administrativo Nacional de Estadísticas (DANE) y cálculos del autor.

PIB: producto interno bruto.

DLGDP: primera diferencia del logaritmo del PIB. 
productos básicos exportables de Colombia (petróleo, carbón, café y níquel) ${ }^{14}$. Se usa la variable DLTотCL (valor unitario de todas las exportaciones colombianas). La segunda definición, obtenida también a través del banco central de Colombia, se denomina тотт y se trata de la relación entre los índices de precios al por mayor de las exportaciones y las importaciones (PX/PI). La variable transformada se denomina DLTOTT (primera diferencia del logaritmo de тотт).

Como diagnóstico gráfico preliminar de la relación clave de este artículo, en los gráficos 3 y 4 se presenta la correlación entre las fluctuaciones de la producción y las variaciones de los términos de intercambio en Colombia. En el gráfico 3 se representa la correlación simple (el coeficiente de correlación es 0,35). En el gráfico 4 se muestra la correlación entre los componentes cíclicos del PIB у тотсL (el coeficiente de correlación es 0,48 ). Además de la correlación positiva, ambos diagramas de dispersión sugieren que estos coeficientes de correlación no aumentan por posibles valores atípicos. En la mayoría de los casos observados en la muestra se sigue el mismo patrón descrito mediante la regresión por mínimos cuadrados ordinarios (MCO) entre DLGDP y DLTOTCL de acuerdo con el gráfico 3 y entre GDPCYCLE у TOTCLCYCLE según el gráfico 4.

Sobre la base del marco de la economía dependiente y la construcción de series relacionadas con los términos de intercambio, en este análisis empírico se supone que los términos de intercambio son exógenos y causantes de las fluctuaciones de la producción, y no lo contrario. A pesar de que se trata de una hipótesis plausible para el caso de Colombia, como se describe en la Introducción, se llevó a cabo una prueba de causalidad de Granger, que sugiere que no debería rechazarse esta suposición (véase el cuadro 1).

Esta prueba de diagnóstico sugiere también que DLTOTCL no es causa a la Granger de DLGDP (aunque los valores $p$ son inferiores a los de la otra hipótesis del cuadro 1). En la siguiente sección se presentarán resultados empíricos más formales sobre la relación entre DLTOTCL y DLGDP. Además de las anteriores definiciones de los términos de intercambio, en el lado derecho de las regresiones se utilizan cuatro precios más relacionados (como alternativa a TOTCL): un índice de Laspeyres para los precios del petróleo, el carbón, el café y el níquel (PXCL), los precios del petróleo (OILPR), el índice de precios al por mayor de las importaciones (PI) y el índice de precios al por mayor de las exportaciones (PX). Las

14 También se calculó un índice de Paasche, pero no presentó una diferencia sustancial con respecto al índice de Laspeyres.
GRÁFICO 3

Correlación de DLGDP y DLTOTCL

(Coeficiente de correlación (simple): 0,35)

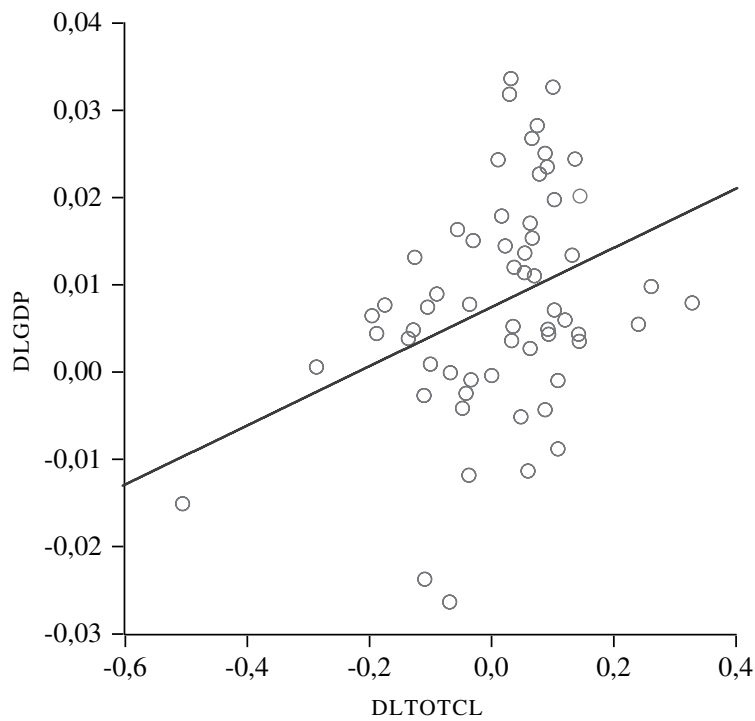

Fuente: Departamento Administrativo Nacional de Estadísticas (DANE), Banco de la República de Colombia (BRC) y cálculos del autor.

Ejes. DLGDP: tasa de crecimiento trimestral del PIB real. DLTOTCL: valor unitario de todas las exportaciones colombianas.

GRÁFICO 4

\section{Correlación de componentes cíclicos del PIB у тотCL \\ (Coeficiente de correlación (simple): 0,48)}

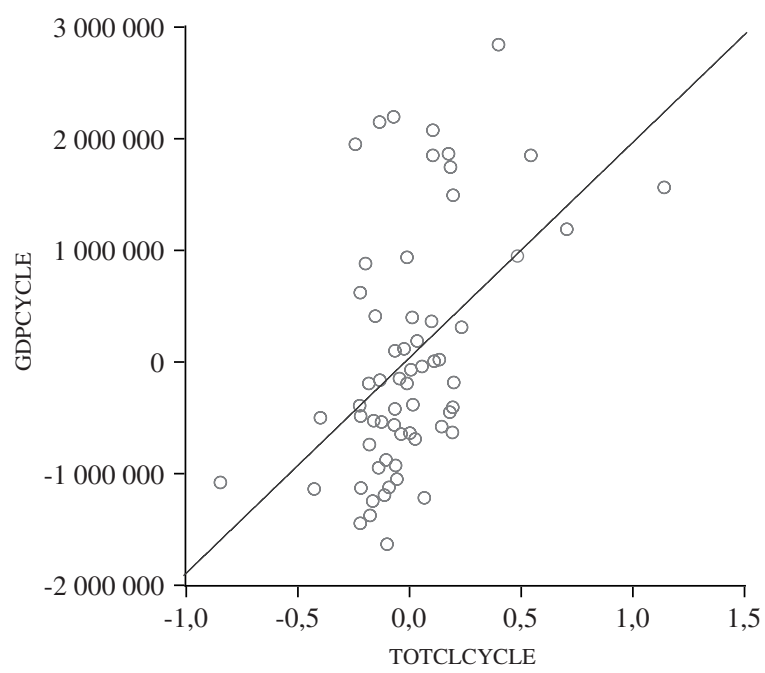

Fuente: Departamento Administrativo Nacional de Estadísticas (DANE), Banco de la República de Colombia (BRC) y cálculos del autor.

PIB: producto interno bruto.

TOTCLCYCLE: componente cíclico de los términos de intercambio de los cuatro productos básicos de exportación. GDPCYCLE: componente cíclico del PIB. 
CUADRO 1

\section{Prueba de causalidad de Granger}

Hipótesis nula: DLGDP no es causa a la Granger de DLTOTCL

\begin{tabular}{|c|c|c|c|c|}
\hline & Longitud del rezago 1 & Longitud del rezago 2 & Longitud del rezago 3 & Longitud del rezago 4 \\
\hline Valor $p$ & 0,80 & 0,79 & 0,82 & 0,52 \\
\hline $\mathrm{N}^{\mathrm{o}}$ de observaciones & 60 & 59 & 58 & 57 \\
\hline \multicolumn{5}{|c|}{ Hipótesis nula: DLTOTCL no es causa a la Granger de DLGDP } \\
\hline Valor $p$ & 0,15 & 0,41 & 0,33 & 0,22 \\
\hline $\mathrm{N}^{\mathrm{o}}$ de observaciones & 60 & 59 & 58 & 57 \\
\hline
\end{tabular}

Fuente: Departamento Administrativo Nacional de Estadísticas (DANE), Banco de la República (BRC) y cálculos del autor.

DLGDP: diferencia del logaritmo del PIB.

DLTOTCL: primera diferencia del logaritmo de TOTCL.

variables transformadas y estacionarias se denominan DLPXCL, DLOILPR, DLPIIFS y DLPXIFS, respectivamente.

- Tasas de interés activas, tipos de cambio, corrientes financieras netas y fluctuaciones de la producción de los Estados Unidos de América

En el lado derecho de la regresión se incluyen cuatro variables de control estacionarias. La primera variable corresponde a la primera diferencia de la tasa de interés activa nominal de los préstamos (DNIR), que se obtuvo de los datos de Estadísticas Financieras Internacionales (IFS, por sus siglas en inglés) y es un promedio ponderado de las tasas efectivas para el conjunto del sistema bancario que abarca todos los tipos de créditos. Como alternativa, se calculó la primera diferencia de la tasa de interés real (DRIR), utilizando la inflación en el índice de precios a la producción.

La segunda variable es la depreciación nominal del tipo de cambio (la primera diferencia del logaritmo del tipo de cambio nominal (DLNER)), que corresponde a la depreciación trimestral del tipo de cambio cuando el valor es positivo. En este caso se utiliza también la primera diferencia del logaritmo del tipo de cambio real (DLRER).

La tercera variable es la primera diferencia de las corrientes financieras netas (las entradas menos las salidas de la balanza de pagos de Colombia). Pese a que las tasas de interés y los tipos de cambio deberían registrar hasta cierto punto el papel de los flujos financieros, esta variable se incluye como posible variable omitida.

Por último, en el análisis econométrico se tiene en cuenta el crecimiento trimestral del PIB de los Estados Unidos de América (DLGDPUs). Esta serie está disponible en las IFS. El mercado estadounidense es destinatario de aproximadamente el $70 \%$ de las exportaciones de petróleo y el $40 \%$ de las exportaciones totales de Colombia.
Las variables de control se pueden correlacionar entre sí. Por ejemplo, cabe la posibilidad de que las alteraciones de la estructura de las tasas de interés, junto con un cierto grado de movilidad del capital, ejerzan presión sobre los tipos de cambio y esa correlación podría afectar a las respectivas pruebas de significación. Sin embargo, la cuestión clave de este artículo es que se produzca un sesgo si estas variables de control se omiten debido a su correlación simultánea con las fluctuaciones de la producción y los términos de intercambio.

\section{Resultados econométricos}

La especificación del componente ARMA del modelo se basaba en el correlograma para la variable dependiente (véase el cuadro A4) y un conjunto de regresiones (véase el cuadro A5) con que se evaluaba la significación de los coeficientes estimados para los elementos del componente ARMA (DLGDP como variable del lado izquierdo). Tanto las funciones de autocorrelación como de correlación parcial sugieren una especificación en torno de ARMA $(3,3)$. Sin embargo, el conjunto de regresiones (incluso un cuarto rezago con una posible interpretación económica) revela una especificación robusta y parsimoniosa. En el cuadro A5 se muestran los resultados de las regresiones, que incluyen lo siguiente: i) solo los elementos autorregresivos (columna 1); ii) solo los elementos del promedio móvil (columna 2); iii) una regresión de base con todos los elementos del componente ARMA (columna 3); iv) una regresión específica obtenida después de quitar uno por uno los elementos cuyos coeficientes estimados no eran significativos al nivel del 5\% en la regresión de base (columna 4), y v) regresiones para los elementos $\mathrm{AR}(2), \mathrm{MA}(3)$ y MA(4) (columnas 5 a 7), que junto con las regresiones de las columnas 1 y 2 muestran que los elementos $\operatorname{Ar}(2)$ y ma(3) son los más robustos. Por lo 
tanto, se utilizó una versión parsimoniosa de ARMA $(2,3)$ sin incluir el primer rezago del componente autorregresivo y sin el primero y segundo rezagos del promedio móvil (columna 8). Las estimaciones de ARMA (2,3) son robustas ante la inclusión de los términos de intercambio y las variables de control. El número de rezagos era razonable para interpretar los efectos en la variable dependiente en el corto plazo. Con los datos trimestrales, el segundo rezago en el componente autorregresivo se refiere a un período de medio año.

En el cuadro 2 (columna 1) se muestran estimaciones positivas y significativas de los coeficientes de ARMA. Mientras que las estimaciones para el coeficiente del promedio móvil pueden estar vinculadas al efecto de las innovaciones estadísticas, la estimación de la parte autorregresiva sugiere la existencia de un importante grado de persistencia en las fluctuaciones del PIB de Colombia. Todas las estimaciones para este modelo ARMA son significativas al menos al nivel del $5 \%$. Con el modelo ARMA se puede explicar un $17 \%$ de la variación total de la variable dependiente. El estadístico $\mathrm{h}$ de Durbin, el valor $p$ de la prueba de chi-cuadrado, la prueba de Breusch-Godfrey para los residuos y el correlograma de los residuos (véase el cuadro A6) indican la ausencia de autocorrelación. Por otra parte, ante el supuesto de estacionaridad débil, DLGDP no enfrenta heteroscedasticidad. Eso significa que los estadísticos $t$ y los valores $p$ utilizados para establecer la significación en el $1,5 \%$ y el $10 \%$ son fiables.

La columna 3 del cuadro A6 corresponde a la regresión que incluye DLTOTCL:

$$
\begin{aligned}
D L G D P_{t}=\alpha & +\gamma_{1} D L T O T C L+\lambda_{2} D L G D P_{t-2} \\
& +\theta_{3} \mu_{t-3}+\mu_{t}
\end{aligned}
$$

La estimación de los efectos de DLTOTCL en DLGDP es significativamente positiva en el nivel del $1 \%$. La magnitud de la estimación del efecto contemporáneo indica que con un aumento del $1 \%$ en el crecimiento de

CUADRO 2

\section{Términos de intercambio y fluctuaciones de la producción (I)}

\begin{tabular}{|c|c|c|c|c|}
\hline & (1) & $(2)$ & (3) & Variable tipificada \\
\hline Constante & $\begin{array}{l}0,0082 * * * \\
(3,00)\end{array}$ & $\begin{array}{l}0,0075^{* * * *} \\
(4,87)\end{array}$ & $\begin{array}{l}0,0082 * * * \\
(2,72)\end{array}$ & \\
\hline $\operatorname{AR}(2)$ & $\begin{array}{l}0,3273 * * * \\
(3,14)\end{array}$ & & $\begin{array}{l}0,3227 * * * \\
(2,96)\end{array}$ & $\begin{array}{l}0,3227 * * * \\
(2,96)\end{array}$ \\
\hline $\operatorname{MA}(3)$ & $\begin{array}{l}0,3377 * * \\
(2,40)\end{array}$ & & $\begin{array}{l}0,3659 * * \\
(2,56)\end{array}$ & $\begin{array}{l}0,3659 * * \\
(2,56)\end{array}$ \\
\hline DLTOTCLt & & $\begin{array}{l}0,0339 \text { *** } \\
(3,95)\end{array}$ & $\begin{array}{l}0,0215 * * * \\
(4,08)\end{array}$ & $\begin{array}{l}0,2315^{* * *} \\
(4,08)\end{array}$ \\
\hline $\begin{array}{l}\text { Efecto total } \\
\text { (persistencia incluida) }\end{array}$ & & & $\begin{array}{l}0,0318 * * * \\
{[10,24]}\end{array}$ & $\begin{array}{l}0,3418^{* * * *} \\
{[10,24]}\end{array}$ \\
\hline$\overline{\mathrm{R}^{2}}$ & 0,17 & 0,12 & 0,26 & 0,26 \\
\hline Valor ajustado de $\mathrm{R}^{2}$ & 0,14 & 0,11 & 0,22 & 0,22 \\
\hline Estadístico de Durbin-Watson & & 1,96 & & \\
\hline Estadístico h de Durbin & 0,71 & & 0,14 & 0,14 \\
\hline Probabilidad chi-cuadrado (Breusch-Godfrey) & 0,20 & & 0,32 & \\
\hline Error estándar de la regresión & 0,01 & & 0,01 & \\
\hline Criterio de información de Akaike & $-6,06$ & & $-6,04$ & \\
\hline Criterio de información de Schwarz & $-5,96$ & & $-5,90$ & \\
\hline Estadístico f (valor $p$ ) & 0,00 & 0,00 & 0,00 & \\
\hline $\mathrm{N}^{\mathrm{o}}$ de observaciones & 67 & 61 & 59 & 59 \\
\hline
\end{tabular}

Variable dependiente: DLGDP (tasa de crecimiento trimestral del PIB real)

Fuente: Departamento Administrativo Nacional de Estadísticas (DANE), Banco de la República (BRC) y cálculos del autor.

Notas:

Estimación por mínimos cuadrados y métodos numéricos.

Errores estándar consistentes entre paréntesis.

$* p<0,10$

$* * p<0,05$

$* * * p<0,01$

Chi-cuadrado entre corchetes.

DLGDP: tasa de crecimiento trimestral del producto interno bruto (PIB) real.

DLTOTCL: primera diferencia del logaritmo de TOTCL. 
los términos de intercambio se incrementa un $0,02 \%$ el crecimiento trimestral del PIB (si se mantienen constantes otras variables). Esta magnitud es importante. Con una desviación típica de DLTOTCL (igual al 13,22\%) variará un $0,28 \%$ el crecimiento trimestral del PIB. Este cambio equivale aproximadamente al $23 \%$ de una desviación típica del crecimiento trimestral del PIв (columna 4 del cuadro A6). Una vez calculado el efecto de la persistencia, la misma desviación típica de DтотLC se vincula a un cambio en torno del $34 \%$ de una desviación típica del crecimiento trimestral del PIB. Por lo tanto, un tercio de la variabilidad trimestral del PIB depende de los términos del intercambio de los cuatro productos básicos más importante de Colombia.

El efecto de los términos de intercambio se mantiene cuando la definición de estos se amplía para incluir el valor unitario de todas las exportaciones colombianas (DLTOTT) (columna 1 del cuadro 3). La estimación es mayor, pero la desviación típica de DLTOTT es menor $(5,79 \%)$. La variable independiente aún representa alrededor del $27 \%$ de una desviación típica del crecimiento del PIB (columna 2 del cuadro 3). El cuadro 3 (columnas 5, 6, 7 y 8 ) y el cuadro 4 (con componentes cíclicos) también ofrecen pruebas que confirman que los precios de los

CUADRO 3

Términos de intercambio y fluctuaciones de la producción II

\begin{tabular}{|c|c|c|c|c|c|c|c|c|c|}
\hline & (1) & $\begin{array}{l}\text { (2) } \\
\text { Variación } \\
\text { estándar }\end{array}$ & (3) & (4) & (5) & (6) & (7) & (8) & $\begin{array}{l}\text { (9) } \\
\text { Variación } \\
\text { estándar }\end{array}$ \\
\hline DLTOTTt & $\begin{array}{l}0,0364 \\
(1,69)\end{array}$ & $\begin{array}{l}0,1715 \\
(1,69)\end{array}$ & & & & & & & \\
\hline DLPXt & & & $\begin{array}{l}0,0253 \\
(1,33)\end{array}$ & & $\begin{array}{l}0,0365 \\
(1,69)\end{array}$ & & & & \\
\hline DLPIt & & & & $\begin{array}{l}0,0028 \\
(0,09)\end{array}$ & $\begin{array}{l}-0,0367 \\
(-1,03)\end{array}$ & & $\begin{array}{l}0,0197 \\
(0,61)\end{array}$ & & \\
\hline DLPXCLt & & & & & & $\begin{array}{l}0,0252 * * * \\
(4,29)\end{array}$ & $\begin{array}{l}0,0260 * * * \\
(3,96)\end{array}$ & & \\
\hline DLOILPRt & & & & & & & & $\begin{array}{l}0,0147 * * * \\
(3,29)\end{array}$ & $\begin{array}{l}0,2043 * * * \\
(3,29)\end{array}$ \\
\hline $\begin{array}{l}\text { Efecto total } \\
\text { (persistencia incluida) }\end{array}$ & $\begin{array}{l}0,0566 \\
{[2,37]}\end{array}$ & $\begin{array}{l}0,2664 \\
{[2,37]}\end{array}$ & & & & $\begin{array}{l}0,0377 * * * \\
{[11,96]}\end{array}$ & & $\begin{array}{l}0,0219 * * * \\
{[7,24]}\end{array}$ & $\begin{array}{l}0,3052 * * * \\
{[7,24]}\end{array}$ \\
\hline$R^{2}$ & 0,20 & 0,20 & 0,19 & 0,17 & 0,20 & 0,27 & 0,28 & 0,25 & 0,25 \\
\hline Valor ajustado de $R^{2}$ & 0,16 & 0,16 & 0,15 & 0,13 & 0,15 & 0,23 & 0,22 & 0,21 & 0,21 \\
\hline Estadístico h de Durbin & 0,15 & 0,15 & 0,47 & 0,75 & 0,08 & 0,28 & 0,50 & 0,65 & 0,65 \\
\hline $\begin{array}{l}\text { Probabilidad chi-cuadrado } \\
\text { (Breusch-Godfrey) }\end{array}$ & 0,22 & & 0,23 & 0,20 & 0,22 & 0,33 & 0,33 & 0,31 & \\
\hline $\begin{array}{l}\text { Error estándar de la regresión } \\
\text { Criterio de información de }\end{array}$ & 0,01 & & 0,01 & 0,01 & 0,01 & 0,01 & 0,01 & 0,01 & \\
\hline Akaike & $-6,07$ & & $-6,06$ & $-6,03$ & $-6,04$ & $-6,06$ & $-6,03$ & $-6,03$ & \\
\hline Criterio de información de & & & & & & & & & \\
\hline Schwarz & $-5,94$ & & $-5,93$ & $-5,90$ & $-5,88$ & $-5,92$ & $-5,85$ & $-5,89$ & \\
\hline Estadístico f (valor $p$ ) & 0,00 & & 0,00 & 0,01 & 0,01 & 0,00 & 0,00 & 0,00 & \\
\hline $\mathrm{N}^{\mathrm{o}}$ de observaciones & 67 & 67 & 67 & 67 & 67 & 59 & 59 & 59 & 59 \\
\hline
\end{tabular}

Fuente: Departamento Administrativo Nacional de Estadísticas (DANE), Banco de la República de Colombia (BRC) y cálculos del autor.

Notas:

Estimación por mínimos cuadrados y métodos numéricos.

Errores estándar consistentes entre paréntesis.

$* p<0,10$

$* * p<0,05$

$* * * p<0,01$

Chi-cuadrado entre corchetes.

El componente de ARMA está incluido en todas las regresiones, pero no se representa.

PIB: producto interno bruto.

DLTOTT: valor unitario de todas las exportaciones colombianas.

DLPX: primera diferencia (trimestre a trimestre) del logaritmo del índice de precios del total de exportaciones (al por mayor).

DLPI: primera diferencia (trimestre a trimestre) del logaritmo del índice de precios del total de importaciones (al por mayor).

DLPXCL: variable transformada y estacionaria.

DLOILPR: variable transformada y estacionaria. 


\begin{tabular}{|c|c|c|c|c|c|c|c|c|c|}
\hline & Estimación & 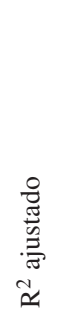 & 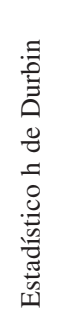 & 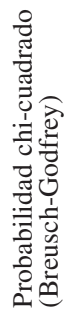 & 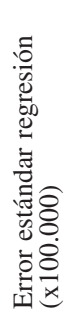 & 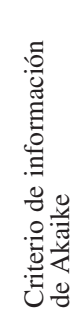 & 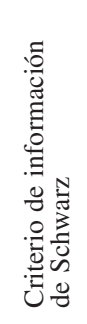 & 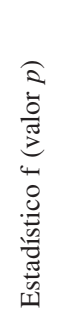 & 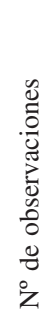 \\
\hline TOTCLt & $\begin{array}{l}542217 * * \\
(2,42)\end{array}$ & 0,75 & 0,59 & 0,80 & 5,7 & 29,41 & 29,58 & 0,00 & 61 \\
\hline Tоттt & $\begin{array}{c}2170543^{* *} \\
(2,36)\end{array}$ & 0,74 & 0,73 & 0,85 & 5,3 & 29,29 & 29,45 & 0,00 & 69 \\
\hline PXt & $\begin{array}{l}14030 \\
(1,95)\end{array}$ & 0,74 & 0,72 & 0,66 & 5,4 & 29,31 & 29,47 & 0,00 & 69 \\
\hline PIt & $\begin{array}{l}-105,4 \\
-(0,01)\end{array}$ & 0,72 & 0,89 & 0,72 & 5,6 & 29,36 & 29,52 & 0,00 & 69 \\
\hline PXCLt & $\begin{array}{l}5928 * * * \\
(3,00)\end{array}$ & 0,74 & 0,53 & 0,75 & 5,6 & 29,39 & 29,56 & 0,00 & 61 \\
\hline OILPRT & $\begin{array}{l}12718^{* *} \\
(2,64)\end{array}$ & 0,74 & 0,59 & 0,75 & 5,7 & 29,41 & 29,6 & 0,00 & 61 \\
\hline
\end{tabular}

Fuente: Departamento Administrativo Nacional de Estadísticas (DANE), Banco de la República de Colombia (BRC) y cálculos del autor.

Notas:

Estimación por mínimos cuadrados y métodos numéricos.

Errores estándar consistentes.

Estadístico t entre corchetes.

$* p<0,10$

$* * p<0,05$

$* * * p<0,01$

$\operatorname{AR}(1) \operatorname{MA}(2)$ y $\operatorname{MA}(3)$ se incluyen, pero no se representan.

TOTCL: índice de precios al por mayor de las importaciones.

тотт: relación entre los índices de precios al por mayor de las exportaciones y las importaciones.

PXt: Índice de precios del total de exportaciones (al por mayor).

PIt: Índice de precios del total de importaciones (al por mayor).

PXCL: precios del petróleo, el carbón, el café y el níquel.

OILPR: precios del petróleo.

cuatro principales bienes de exportación de Colombia, en particular el petróleo, son los que tienen una mayor incidencia en la producción en el corto plazo.

\section{Pruebas de robustez}

Los resultados de la sección III.2 son robustos ante la inclusión de las variables de control tasas de interés de los préstamos, tipos de cambio, corrientes financieras netas y crecimiento trimestral del PIB de los Estados Unidos de América (véase el cuadro 5). La estimación para DLTOTCL no solo sigue siendo significativa al $1 \%$ en la mayoría de las regresiones (al 5\% en la columna 4), sino que además su magnitud es estable.
Para exponer los resultados a una prueba de robustez más estricta, se incluyeron variables de control desfasadas que eran independientemente significativas cuando se ejecutó una regresión para DLGDP. Las variables son las siguientes: DNIR y DLGDPUS (ambas con un rezago de dos trimestres). Una vez incluidas estas variables, solo DNIR (-2) sigue siendo significativa (columnas 1 y 3 del cuadro 6). Las estimaciones para DLTOTCL tipificada siguen siendo robustas y el efecto total, incluida la persistencia, representa el $30 \%$ de variabilidad del PIB (columna 4 del cuadro 6).

En los resultados de las variables tipificadas (columna 4 del cuadro 6) también se registra un efecto negativo teóricamente sistemático de DNIR (-2), que 
CUADRO 5

\section{Robustez ante variables adicionales (I)}

Variable dependiente: DLGDP (tasa de crecimiento trimestral del PIB real)

\begin{tabular}{|c|c|c|c|c|c|c|c|}
\hline & {$[1]$} & {$[2]$} & {$[3]$} & [4] & {$[5]$} & {$[6]$} & [7] \\
\hline Constante & $\begin{array}{l}0,0082 * * * \\
(2,72)\end{array}$ & $\begin{array}{l}0,0082 * * * \\
(2,72)\end{array}$ & $\begin{array}{l}0,0081 \text { **** } \\
(2,70)\end{array}$ & $\begin{array}{l}0,0079 * * \\
(2,60)\end{array}$ & $\begin{array}{l}0,0081 * * * \\
(2,71)\end{array}$ & $\begin{array}{l}0,0086^{* * * *} \\
(3,07)\end{array}$ & $\begin{array}{l}0,0078 * * \\
(2,66)\end{array}$ \\
\hline $\operatorname{AR}(2)$ & $\begin{array}{l}0,3227 * * * \\
(2,96)\end{array}$ & $\begin{array}{l}0,3204 * * \\
(2,29)\end{array}$ & $\begin{array}{l}0,3231 \text { **** } \\
(2,90)\end{array}$ & $\begin{array}{l}0,3356^{* * * *} \\
(3,12)\end{array}$ & $\begin{array}{l}0,3241 * * * \\
(3,06)\end{array}$ & $\begin{array}{l}0,3105^{* * * *} \\
(3,00)\end{array}$ & $\begin{array}{l}0,2855^{* * *} \\
(2,44)\end{array}$ \\
\hline MA(3) & $\begin{array}{l}0,3659 * * \\
(2,56)\end{array}$ & $\begin{array}{l}0,3624 * * \\
(2,19)\end{array}$ & $\begin{array}{l}0,3626 * * \\
(2,50)\end{array}$ & $\begin{array}{l}0,3657^{* *} \\
(2,52)\end{array}$ & $\begin{array}{l}0,3581 * * * \\
(2,53)\end{array}$ & $\begin{array}{l}0,3627 * * \\
(2,42)\end{array}$ & $\begin{array}{l}0,3843^{* * *} \\
(2,58)\end{array}$ \\
\hline DLTOTCLt & $\begin{array}{l}0,0215 * * * \\
(4,08)\end{array}$ & $\begin{array}{l}0,0221 * * * \\
(3,86)\end{array}$ & $\begin{array}{l}0,0217 * * * \\
(4,11)\end{array}$ & $\begin{array}{l}0,0279 * * \\
(2,57)\end{array}$ & $\begin{array}{l}0,0226 * * * \\
(4,02)\end{array}$ & $\begin{array}{l}0,0237 * * * \\
(2,69)\end{array}$ & $\begin{array}{l}0,0201 * * * \\
(3,63)\end{array}$ \\
\hline DNIRt & & $\begin{array}{l}0,0144 \\
(0,23)\end{array}$ & & & & & \\
\hline DRIRt & & & $\begin{array}{l}-0,0008 \\
(-0,29)\end{array}$ & & & & \\
\hline DLNERT & & & & $\begin{array}{l}0,0234 \\
(0,77)\end{array}$ & & & \\
\hline DLRERt & & & & & $\begin{array}{l}0,0127 \\
(0,56)\end{array}$ & & \\
\hline DLGDPUS & & & & & & $\begin{array}{l}-0,0878 \\
(-0,32)\end{array}$ & \\
\hline DNFF & & & & & & & $\begin{array}{l}0,0000 \\
(0,48)\end{array}$ \\
\hline$R^{2}$ & 0,26 & 0,26 & 0,26 & 0,27 & 0,26 & 0,26 & 0,26 \\
\hline Valor ajustado de $R^{2}$ & 0,22 & 0,21 & 0,20 & 0,21 & 0,21 & 0,21 & 0,20 \\
\hline Estadístico h de Durbin & 0,14 & $\ldots$ & 0,15 & 0,27 & 0,26 & 0,25 & 0,41 \\
\hline Probabilidad chi-cuadrado (Breusch-Godfrey) & 0,32 & 0,29 & 0,34 & 0,36 & 0,33 & 0,29 & 0,35 \\
\hline Error estándar de la regresión & 0,01 & 0,01 & 0,01 & 0,01 & 0,01 & 0,01 & 0,01 \\
\hline Criterio de información de Akaike & $-6,04$ & $-6,00$ & $-6,01$ & $-6,02$ & $-6,01$ & $-6,01$ & $-6,00$ \\
\hline Criterio de información de Schwarz & $-5,90$ & $-5,83$ & $-5,94$ & $-5,84$ & $-5,84$ & $-5,83$ & $-5,93$ \\
\hline Estadístico f (valor $p$ ) & 0,00 & 0,00 & 0,00 & 0,00 & 0,00 & 0,00 & 0,00 \\
\hline $\mathrm{N}^{\mathrm{o}}$ de observaciones & 59 & 59 & 59 & 59 & 59 & 59 & 58 \\
\hline
\end{tabular}

Fuente: Departamento Administrativo Nacional de Estadísticas (DANE), Banco de la República de Colombia (BRC) y cálculos del autor.

Notas:

PIB: producto interno bruto.

Estimación por mínimos cuadrados y métodos numéricos.

Errores estándar consistentes.

Estadístico t entre paréntesis.

$* p<0,10$

$* * p<0,05$

$* * * p<0,01$

DLTOTCL: valor unitario de todas las exportaciones colombianas.

DNIR: tasa de interés activa nominal de los préstamos.

DRIR: primera diferencia de la tasa de interés real.

DLNER: primera diferencia del logaritmo del tipo de cambio nominal.

DLRER: primera diferencia del logaritmo del tipo de cambio real.

DLGDPUS: crecimiento trimestral del PIB de los Estados Unidos.

DNFF: Primera diferencia trimestre a trimestre de flujos financieros netos. 
CUADRO 6

Robustez ante variables adicionales (II)

(Incluidos los retardos)

Variable dependiente: DLGDP (tasa de crecimiento trimestral del PIB real)

\begin{tabular}{|c|c|c|c|c|c|c|}
\hline & (1) & (2) & (3) & $\begin{array}{l}\text { (4) } \\
\text { Variación } \\
\text { típica }\end{array}$ & $\begin{array}{c}(5) \\
\text { No se incluye } \\
\text { ARMA }\end{array}$ & $\begin{array}{c}(6) \\
\text { No se incluye } \\
\text { ARMA } \\
\text { Variación típica }\end{array}$ \\
\hline DLTOTCLt & $\begin{array}{l}0,0182 * * * \\
(3,81)\end{array}$ & $\begin{array}{l}0,0189 * * * \\
(3,08)\end{array}$ & $\begin{array}{l}0,0146^{* *} \\
(2,33)\end{array}$ & $\begin{array}{l}0,1961 * * * \\
(3,81)\end{array}$ & $\begin{array}{l}0,0319 * * * \\
(4,05)\end{array}$ & $\begin{array}{l}0,3432 * * * \\
(4,05)\end{array}$ \\
\hline DNIRt-2 & $\begin{array}{l}-0,1777 * * \\
(-2,61)\end{array}$ & & $\begin{array}{l}-0,1813 \text { *** } \\
(-2,72)\end{array}$ & $\begin{array}{l}-0,3552 * * \\
(-2,61)\end{array}$ & $\begin{array}{l}-0,1251 \\
(-1,28)\end{array}$ & $\begin{array}{l}-0,2500 \\
(-1,28)\end{array}$ \\
\hline DLGDPUSt- 2 & & $\begin{array}{l}-0,1762 \\
(-1,19)\end{array}$ & $\begin{array}{l}-0,2637 \\
(-1,61)\end{array}$ & & & \\
\hline $\begin{array}{l}\text { Efecto total DLTOTCL } \\
\text { (persistencia incluida) }\end{array}$ & & & & $\begin{array}{l}0,3076 * * * \\
{[8,46]}\end{array}$ & & \\
\hline $\begin{array}{l}\text { Efecto total DNIRt-2 } \\
\text { (persistencia incluida) }\end{array}$ & & & & $\begin{array}{l}-0,5573 * * \\
{[4,96]}\end{array}$ & & \\
\hline$\overline{R^{2}}$ & 0,39 & 0,27 & 0,41 & 0,39 & 0,18 & 0,18 \\
\hline Valor ajustado de $R^{2}$ & 0,34 & 0,21 & 0,35 & 0,34 & 0,15 & 0,15 \\
\hline Estadístico de Durbin-Watson & & & & & 2,09 & 2,09 \\
\hline Estadístico h de Durbin & $-2,33$ & 0,34 & $-1,77$ & $-1,77$ & & \\
\hline Probabilidad chi-cuadrado (Breusch-Godfrey) & 0,43 & 0,24 & 0,42 & & & \\
\hline Error estándar de la regresión & 0,01 & 0,01 & 0,01 & & 0,01 & \\
\hline Criterio de información de Akaike & $-6,20$ & $-6,02$ & $-6,19$ & & & \\
\hline Criterio de información de Schwarz & $-6,02$ & $-5,84$ & $-5,98$ & & & \\
\hline Estadístico f (valor $p$ ) & 0,00 & 0,00 & 0,00 & 0,00 & 0,00 & 0,00 \\
\hline $\mathrm{N}^{\circ}$ de observaciones & 59 & 59 & 59 & 59 & 61 & 61 \\
\hline
\end{tabular}

Fuente: Departamento Administrativo Nacional de Estadísticas (DANE), Banco de la República de Colombia (BRC), Estadísticas Financieras Internacionales y cálculos del autor.

Notas:

PIB: producto interno bruto.

Estimación por mínimos cuadrados y métodos numéricos.

Errores estándar consistentes.

Estadístico t entre paréntesis.

Chi-cuadrado entre corchetes.

$* p<0,10$

$* * p<0,05$

$* * * x<0,01$

Se incluye el componente ARMA, pero no se representa en las regresiones (1) a (4).

DLTOTCL: valor unitario de todas las exportaciones colombianas.

DNIR: tasa de interés activa nominal de los préstamos.

DLGDPUS: crecimiento trimestral del PIB de los Estados Unidos.

es significativo e importante en magnitud. A pesar de que la estimación no es robusta cuando se quitan los componentes de ARMA, con la inclusión de DNIR (-2) aumenta $R^{2}$ de 0,17 a 0,39 . Si bien es cierto que la finalidad de este artículo no es evaluar las propiedades del modelo para realizar pronósticos, ni la robustez de la estimación del efecto de DNIR (-2), la estimación negativa, junto con un valor de $R^{2}$ mayor puede reflejar el hecho de que DNIR está actuando a través de la inversión, que es el componente de la demanda agregada cuyas variaciones explican la mayoría de las variaciones del PIB en el corto plazo. Aunque la inversión representa una cuarta parte del PIB de Colombia, mientras que el consumo alcanza dos tercios, la primera constituye el componente más volátil del PIB (su desviación típica es ocho veces mayor que en el caso del consumo). Una exploración inicial de los canales de la demanda agregada relevantes para entender mejor el efecto significativo y robusto de los términos de intercambio en las fluctuaciones de la producción en Colombia (véase el cuadro 7) indica que la inversión (DIL) es el canal principal. Una desviación típica en DLTOTCL parece explicar un tercio de la variabilidad de la inversión (solo para el efecto contemporáneo). 
CUADRO 7

Términos de intercambio y componentes de la demanda agregada

(Regresiones de mínimos cuadrados ordinarios (MCO))

\begin{tabular}{|c|c|c|c|c|c|c|}
\hline & (1) & (2) & (3) & (4) & (5) & (6) \\
\hline Variable dependiente: & DLC & DLI & DLG & DLX & DLM & DLI \\
\hline Constante & $\begin{array}{l}0,0068 * * * \\
(4,53)\end{array}$ & $\begin{array}{l}0,0086 \\
(0,82)\end{array}$ & $\begin{array}{l}0,011 \text { **** } \\
(4,18)\end{array}$ & $\begin{array}{l}0,0099 * * \\
(2,36)\end{array}$ & $\begin{array}{l}0,0129 \text { ** } \\
(2,04)\end{array}$ & $\begin{array}{l}0,0037 \\
(0,36)\end{array}$ \\
\hline DLTOTCLt & $\begin{array}{l}0,0130 \\
(1,36)\end{array}$ & $\begin{array}{l}0,2096 \text { *** } \\
(3,30)\end{array}$ & $\begin{array}{l}0,0330 \text { ** } \\
(2,22)\end{array}$ & $\begin{array}{l}-0,0296 \\
(-0,74)\end{array}$ & $\begin{array}{l}0,0972 \\
(1,65)\end{array}$ & $\begin{array}{l}0,1939 \text { **** } \\
(3,22)\end{array}$ \\
\hline DNIRt-2 & & & & & & $\begin{array}{l}-1,0042 \\
(-1,65)\end{array}$ \\
\hline$\overline{R^{2}}$ & 0,02 & 0,10 & 0,04 & 0,01 & 0,06 & 0,19 \\
\hline Valor ajustado de $R^{2}$ & 0,004 & 0,09 & 0,03 & $-0,003$ & 0,05 & 0,16 \\
\hline Estadístico de Durbin-Watson & 1,33 & 2,05 & 1,93 & 1,98 & 1,42 & 2,25 \\
\hline Error estándar de la regresión & 0,01 & 0,09 & 0,03 & 0,03 & 0,05 & 0,08 \\
\hline Estadístico $\mathrm{f}$ (valor $p$ ) & 0,26 & 0,01 & 0,11 & 0,37 & 0,05 & 0,00 \\
\hline $\mathrm{N}^{\mathrm{o}}$ de observaciones & 61 & 61 & 61 & 61 & 61 & 61 \\
\hline
\end{tabular}

Fuente: Departamento Administrativo Nacional de Estadísticas (DANE), Banco de la República de Colombia, Estadísticas Financieras Internacionales y cálculos del autor.

Notas:

Estadístico t entre paréntesis.

$* \mathrm{p}<0,10$

$* * \mathrm{p}<0,05$

$* * * \mathrm{p}<0,01$

DLI: primera diferencia (trimestre a trimestre) del logaritmo de la inversión agregada real.

DLC: primera diferencia (trimestre a trimestre) del logaritmo de consumo agregado real.

DLG: gasto público.

DLX: exportaciones.

DLM: importaciones.

DLTOTCL: valor unitario de todas las exportaciones colombianas.

DNIR: tasa de interés activa nominal de los préstamos.

Las investigaciones futuras se orientarán a examinar qué tipo de inversiones están fomentando en el corto plazo las fluctuaciones de los términos de intercambio. Esto también podría requerir una mejor comprensión de los mecanismos a través de los cuales los términos de intercambio pueden afectar a los mercados crediticios y a las tasas de interés. El siguiente canal que sugiere el cuadro 7, pero que aparentemente resulta menos sensible, es el gasto público (DLG). Por último, la cuenta corriente (DLX para las exportaciones y DLM para las importaciones) no parece estar en correlación con los términos de intercambio en el corto plazo. 


\section{IV}

\section{Observaciones finales}

Aunque a veces se afirma que una correlación positiva entre los términos de intercambio y la producción agregada se puede establecer a priori, en la literatura hay numerosos estudios que describen la complejidad de la relación entre estas dos variables. En primer lugar, en algunos países en desarrollo se ha detectado una correlación positiva y otra negativa. Segundo, el marco teórico habitualmente utilizado para la descripción de las pequeñas economías abiertas permite resultados en que la relación puede ser negativa o nula. Mucho depende de la plausibilidad de las hipótesis formuladas para una economía determinada y de la forma en que los mercados nacionales se ajustan después de que se produzcan perturbaciones externas. La idea de un efecto ambiguo se ha incorporado recientemente en Colombia al debate sobre las consecuencias perversas de los términos de intercambio y la bien conocida enfermedad holandesa. Este resultado, que normalmente se vincula al largo plazo, también podría producirse en el corto plazo en función de la rapidez con que se transmitan los efectos contractivos potenciales de un auge de precios de los productos básicos.

La estimación de la repercusión en el PIB de los términos de intercambio no solo resultó ser significativamente positiva, sino también de magnitud muy considerable. Una desviación típica en el crecimiento de los términos de intercambio se traduce en alrededor un tercio de una desviación típica del crecimiento del PIB trimestral. Los resultados son robustos ante diferentes especificaciones, como los componentes de precios de los términos de intercambio, definiciones alternativas de los ciclos económicos y variables de control cuya omisión podría dar lugar a una estimación sesgada.

Además, la depreciación del tipo de cambio nominal no parece tener un efecto muy significativo en el corto plazo, como se afirma en la hipótesis de la devaluación contractiva, lo que podría ser importante a la hora de analizar posibles nuevas políticas - costosas o distorsionadoras - orientadas al control de la apreciación del tipo de cambio nominal, que últimamente han experimentado Colombia y otros países en desarrollo. Asimismo, esta dinámica de corto plazo podría complementar el análisis que sugiere que la devaluación es una herramienta útil para el crecimiento. Por otra parte, en este estudio se han hallado pruebas preliminares que apoyan la creencia de que la tasa de interés activa puede tener un efecto negativo en las fluctuaciones de la producción.

En resumen, se encontró evidencia empírica sólida que respalda la hipótesis de que los términos de intercambio han desempeñado un papel importante en la determinación de las variaciones de corto plazo en el PIB de Colombia durante el período 1994-2011. Los resultados de especificaciones simples de series estacionarias, que se justifican con pruebas de series cronológicas (pruebas de cointegración y de causalidad de Granger), junto con las características particulares de la economía colombiana, sugieren que los términos de intercambio son exógenos y una fuente de las fluctuaciones de la producción según la descripción del modelo de tres tipos de bienes para una economía dependiente. Las pruebas no indican - por lo menos en el corto plazo- que los posibles efectos negativos de los términos de intercambio (enfermedad holandesa), en caso de existir, puedan contrarrestar los efectos positivos en la producción agregada.

La información empírica preliminar indica también que la inversión puede ser el principal componente de la demanda que determina el resultado agregado. Una limitación de este estudio radica en el uso de datos agregados. Por lo tanto, una futura extensión consistiría en examinar la relación entre los términos de intercambio y la demanda de inversiones desagregada por componentes y por sectores industriales, con el fin de determinar el fundamento del rápido ajuste observado de los shocks externos en el corto plazo. En futuras investigaciones relacionadas con este hallazgo también se explorarán los efectos a corto plazo de las fluctuaciones de los términos de intercambio en los mercados crediticios, las tasas de interés y la inversión en Colombia. Estos estudios permitirían una evaluación más detallada de los mecanismos que operan en las rápidas respuestas de la inversión y la producción a las turbulencias externas. 
ANEXO

CUADRO A1

Resumen de pruebas de cointegración (PIB y TOTCL)

Muestra: trim 4 de 1993 y trim 2 de 2011

Observaciones incluidas: 59

Serie: TOTCL GDP

Intervalo de rezagos: 1 a 2

Selección (nivel 0,05*) Número de relaciones de cointegración por modelo

\begin{tabular}{|c|c|c|c|c|c|}
\hline Tendencia de los datos: & Ninguna & Ninguna & Lineal & Lineal & Cuadrática \\
\hline Tipo de prueba & Sin intersección & Intersección & Intersección & Intersección & Intersección \\
\hline & Ninguna tendencia & Ninguna tendencia & Ninguna tendencia & Tendencia & Tendencia \\
\hline Traza & 0 & 0 & 0 & 0 & 0 \\
\hline $\begin{array}{l}\text { Prueba de máximo } \\
\text { autovalor (Max-eig.) }\end{array}$ & 0 & 0 & 0 & 0 & 0 \\
\hline
\end{tabular}

* Valores críticos basados en MacKinnon, Haug y Michelis (1999)

Criterios de información por rango y modelo

\begin{tabular}{|c|c|c|c|c|c|}
\hline Tendencia de los datos: & Ninguna & Ninguna & Lineal & Lineal & Cuadrática \\
\hline Rango o & Sin intersección & Intersección & Intersección & Intersección & Intersección \\
\hline $\begin{array}{l}\mathrm{N}^{\mathrm{o}} \text { de ecuaciones de } \\
\text { cointegración }\end{array}$ & Ninguna tendencia & Ninguna tendencia & Ninguna tendencia & Tendencia & Tendencia \\
\hline \multicolumn{6}{|c|}{ Logaritmo de verosimilitud por rango (filas) y modelo (columnas) } \\
\hline 0 & $-864,2456$ & $-864,2456$ & $-860,4798$ & $-860,4798$ & $-858,4805$ \\
\hline 1 & $-860,1549$ & $-859,1981$ & $-856,0961$ & $-854,2298$ & $-852,4016$ \\
\hline 2 & $-859,5365$ & $-855,9931$ & $-855,9931$ & $-851,0928$ & $-851,0928$ \\
\hline \multicolumn{6}{|c|}{ Logaritmo de verosimilitud por rango (filas) y modelo (columnas) } \\
\hline 0 & 29,56765 & 29,56765 & 29,50779 & 29,50779 & 29,50781 \\
\hline 1 & 29,56457 & 29,56604 & 29,49478 & 29,46542 & 29,43734 \\
\hline 2 & 29,6792 & 29,62689 & 29,62689 & 29,52857 & 29,52857 \\
\hline \multicolumn{6}{|c|}{ Criterio de información de Schwarz por rango (filas) y modelo (columnas) } \\
\hline 0 & 29,84935 & 29,84935 & 29,85991 & 29,85991 & 29,93036 \\
\hline 1 & 29,98712 & 30,0238 & 29,98776 & 29,99361 & 30,00074 \\
\hline 2 & 30,2426 & 30,26071 & 30,26071 & 30,23282 & 30,23282 \\
\hline
\end{tabular}

Fuente: Departamento Administrativo Nacional de Estadísticas (DANE), Banco de la República de Colombia (BRC) y cálculos del autor. 
CUADRO A2

Datos y definición de la muestra

\begin{tabular}{|c|c|c|c|}
\hline Código & Definición & Fuente & Alcance \\
\hline$\underline{\mathrm{D}}$ & Primera diferencia trimestre a trimestre de... & & \\
\hline DL & Primera diferencia (trimestre a trimestre) del logaritmo de... & & \\
\hline GDP & Producto interno bruto (PIB) real & $\begin{array}{l}\text { Departamento Administrativo } \\
\text { Nacional de Estadísticas (DANE) }\end{array}$ & 1994 trim I - 2011 trim Il \\
\hline TотCL & $\begin{array}{l}\text { Términos de intercambio de cuatro productos básicos } \\
\text { (petróleo, carbón, café y níquel) }\end{array}$ & $\begin{array}{l}\text { Banco de la República, cálculos } \\
\text { del autor }\end{array}$ & 1996 trim I - 2011 trim Il \\
\hline тотт & $\begin{array}{l}\text { Términos de intercambio (precios al por mayor de } \\
\text { exportaciones e importaciones) }\end{array}$ & Banco de la República & 1994 trim I - 2011 trim Il \\
\hline$\underline{\mathrm{PX}}$ & Índice de precios del total de exportaciones (al por mayor) & Banco de la República & 1994 trim I - 2011 trim II \\
\hline$\underline{\text { PI }}$ & Índice de precios del total de importaciones (al por mayor) & Banco de la República & 1994 trim I - 2011 trim II \\
\hline PXCL & $\begin{array}{l}\text { Índice de precios de Laspeyres para las exportaciones de } \\
\text { cuatro productos básicos (petróleo, carbón, café y níquel) }\end{array}$ & $\begin{array}{l}\text { Banco de la República, cálculos } \\
\text { del autor }\end{array}$ & 1996 trim I - 2011 trim II \\
\hline OILPR & Índice de precios de Laspeyres para el petróleo & $\begin{array}{l}\text { Banco de la República, cálculos } \\
\text { del autor }\end{array}$ & 1996 I - 2011 II \\
\hline NIR & Tasa de interés activa nominal & $\begin{array}{l}\text { Estadísticas Financieras } \\
\text { Internacionales (IFE) }\end{array}$ & 1994 trim I - 2011 trim Il \\
\hline RIR & $\begin{array}{l}\text { Tasa de interés real activa (utilizando la inflación del índice } \\
\text { de precios a la producción) }\end{array}$ & $\begin{array}{l}\text { Estadísticas Financieras } \\
\text { Internacionales, cálculos del } \\
\text { autor }\end{array}$ & 1994 I - 2011 II \\
\hline NER & $\begin{array}{l}\text { Tipo de cambio nominal (pesos por dólares de los } \\
\text { Estados Unidos) }\end{array}$ & Banco de la República & 1994 trim I - 2011 trim Il \\
\hline$\underline{\text { RER }}$ & Tipo de cambio real (índice de precios a la producción) & Banco de la República & 1994 trim I - 2011 trim Il \\
\hline GDPUS & PIB real de los Estados Unidos & $\begin{array}{l}\text { Estadísticas Financieras } \\
\text { Internacionales }\end{array}$ & 1994 trim I - 2011 trim Il \\
\hline$\underline{\mathrm{NFF}}$ & Flujos financieros netos (entradas-salidas) & Banco de la República & 1996 trim I - 2011 trim Il \\
\hline $\mathrm{C}$ & Consumo agregado real & $\begin{array}{l}\text { Departamento Administrativo } \\
\text { Nacional de Estadísticas (DANE) }\end{array}$ & 1994 trim I - 2011 trim Il \\
\hline I & Inversión agregada real & $\begin{array}{l}\text { Departamento Administrativo } \\
\text { Nacional de Estadísticas (DANE) }\end{array}$ & 1994 trim I - 2011 trim Il \\
\hline G & Gasto público agregado real & $\begin{array}{l}\text { Departamento Administrativo } \\
\text { Nacional de Estadísticas (DANE) }\end{array}$ & 1994 trim I - 2011 trim Il \\
\hline $\mathrm{x}$ & Exportaciones reales & $\begin{array}{l}\text { Departamento Administrativo } \\
\text { Nacional de Estadísticas (DANE) }\end{array}$ & 1994 trim I - 2011 trim Il \\
\hline M & Importaciones reales & $\begin{array}{l}\text { Departamento Administrativo } \\
\text { Nacional de Estadísticas (DANE) }\end{array}$ & 1994 trim I - 2011 trim Il \\
\hline
\end{tabular}

Fuente: Departamento Administrativo Nacional de Estadísticas (DANE), Banco de la República de Colombia (BRC), Estadísticas Financieras Internacionales y cálculos del autor. 
CUADRO A3

Pruebas de raíces unitarias Dickey-Fuller aumentadas

Valores $p$ de MacKinnon unilaterales

\begin{tabular}{|c|c|}
\hline Variable & Hipótesis nula: la variable tiene una raíz unitaria \\
\hline DLGDP & 0,002 \\
\hline DLTOTCL & 0,000 \\
\hline DLTOTT & 0,000 \\
\hline DLPX & 0,000 \\
\hline DLPI & 0,000 \\
\hline DLPXCL & 0,000 \\
\hline DLOILPR & 0,000 \\
\hline GDP (CYCLE) & 0,001 \\
\hline TOTCL (CYCLE) & 0,000 \\
\hline TOTT (CYCLE) & 0,000 \\
\hline PX (CYCLE) & 0,000 \\
\hline PI (CYCLE) & 0,008 \\
\hline PXCL (CYCLE) & 0,000 \\
\hline OILPR (CYCLE) & 0,000 \\
\hline DNIR & 0,000 \\
\hline DRIR & 0,000 \\
\hline DLNER & 0,000 \\
\hline DLRER & 0,000 \\
\hline DLGDPUS & 0,000 \\
\hline DNFF & 0,000 \\
\hline DLC & 0,000 \\
\hline DLI & 0,000 \\
\hline DLG & 0,000 \\
\hline DLX & 0,000 \\
\hline DLM & 0,000 \\
\hline
\end{tabular}

Fuente: Departamento Administrativo Nacional de Estadísticas (DANE), Banco de la República de Colombia (BRC), Estadísticas Financieras Internacionales y cálculos del autor.

Nota: Criterio de información de Schwarz.

DLGDP: tasa de crecimiento trimestral del PIB real.

DLTOTCL: valor unitario de todas las exportaciones colombianas.

DLтотт: primera diferencia del logaritmo de тотт.

DLPX: primera diferencia (trim a trim) del logaritmo del índice de precios del total de exportaciones (al por mayor). DLPI: primera diferencia (trim a trim) del logaritmo del índice de precios del total de importaciones (al por mayor).

DLPXCL: variable transformada y estacionaria.

DLOILPR: variable transformada y estacionaria.

GDPCYCLE: componente cíclico del PIB.

TOTCLCYCLE: componente cíclico de los términos de intercambio de los cuatro productos básicos de exportación.

тот: relación entre los índices de precios al por mayor de las exportaciones y las importaciones.

PI: índice de precios al por mayor de las importaciones.

PX : índice de precios al por mayor de las exportaciones.

PXCL: precios del petróleo, el carbón, el café y el níquel.

OILPR: precios del petróleo.

DNIR: tasa de interés activa nominal de los préstamos.

DRIR: primera diferencia de la tasa de interés real.

DLNER: primera diferencia del logaritmo del tipo de cambio nominal.

DLRER: primera diferencia del logaritmo del tipo de cambio real.

DLGDPUS: crecimiento trimestral del PIB de los Estados Unidos de América.

DLG: gasto público.

DLX: exportaciones.

DLM: importaciones. 
CUADRO A4

ARMA específico

(Correlograma para DLGDP)

\begin{tabular}{|c|c|c|c|c|}
\hline Rezago & Autocorrelación & Correlación parcial & Estadístico Q & Probabilidad de estadístico Q \\
\hline 1 & 0,09 & 0,09 & 0,52 & 0,47 \\
\hline 2 & 0,30 & 0,30 & 7,17 & 0,03 \\
\hline 3 & 0,24 & 0,22 & 11,48 & 0,01 \\
\hline 4 & $-0,07$ & $-0,20$ & 11,82 & 0,02 \\
\hline 5 & $-0,04$ & $-0,20$ & 11,93 & 0,04 \\
\hline 6 & 0,00 & 0,04 & 11,93 & 0,06 \\
\hline 8 & $-0,10$ & $-0,09$ & 12,88 & 0,12 \\
\hline 9 & 0,19 & 0,09 & 15,78 & 0,07 \\
\hline 10 & 0,01 & 0,00 & 15,78 & 0,11 \\
\hline 11 & $-0,03$ & $-0,07$ & 15,83 & 0,15 \\
\hline 12 & 0,11 & 0,03 & 16,93 & 0,15 \\
\hline 13 & 0,00 & 0,07 & 16,93 & 0,20 \\
\hline 14 & $-0,08$ & $-0,09$ & 17,50 & 0,23 \\
\hline 15 & 0,02 & $-0,05$ & 17,52 & 0,29 \\
\hline 17 & $-0,01$ & 0,14 & 18,48 & 0,36 \\
\hline 18 & $-0,20$ & $-0,23$ & 22,24 & 0,22 \\
\hline 19 & 0,03 & 0,01 & 22,30 & 0,27 \\
\hline 20 & $-0,07$ & 0,08 & 22,78 & 0,30 \\
\hline 21 & $-0,11$ & $-0,05$ & 23,96 & 0,30 \\
\hline 22 & 0,04 & $-0,08$ & 24,09 & 0,34 \\
\hline 23 & $-0,14$ & $-0,07$ & 26,06 & 0,30 \\
\hline 24 & $-0,02$ & 0,03 & 26,11 & 0,35 \\
\hline 25 & $-0,08$ & 0,02 & 26,86 & 0,36 \\
\hline 26 & $-0,01$ & $-0,03$ & 26,87 & 0,42 \\
\hline 27 & $-0,12$ & $-0,04$ & 28,57 & 0,38 \\
\hline 28 & $-0,05$ & $-0,07$ & 28,81 & 0,42 \\
\hline
\end{tabular}

Fuente: Departamento Administrativo Nacional de Estadísticas (DANE) y cálculos del autor. 
CUADRO A5

ARMA específico

(Regresiones para componentes de ARMA)

Variable dependiente: DLGDP (tasa de crecimiento trimestral del PIB real)

\begin{tabular}{|c|c|c|c|c|c|c|c|c|}
\hline & (1) & (2) & (3) & $\begin{array}{c}\text { (4) } \\
\text { Específicación } \\
\text { I }\end{array}$ & (5) & (6) & (7) & $\begin{array}{c}\text { (8) } \\
\text { Específicación } \\
\text { II }\end{array}$ \\
\hline Constante & $\begin{array}{l}0,0081 \text { *** } \\
(3,33)\end{array}$ & $\begin{array}{l}0,0085^{* * * *} \\
(3,82)\end{array}$ & $\begin{array}{l}0,0082 * * * \\
(3,27)\end{array}$ & $\begin{array}{l}0,0082 * * * \\
(3,48)\end{array}$ & $\begin{array}{l}0,0079 * * * \\
(3,80)\end{array}$ & $\begin{array}{l}0,0085 * * * \\
(4,43)\end{array}$ & $\begin{array}{l}0,0082 * * * \\
(6,08)\end{array}$ & $\begin{array}{l}0,0082 * * * \\
(3,00)\end{array}$ \\
\hline $\operatorname{AR}(1)$ & $\begin{array}{l}0,0498 \\
(0,33)\end{array}$ & & $\begin{array}{l}-0,5984 \\
(-1,89)\end{array}$ & & & & & \\
\hline $\operatorname{AR}(2)$ & $\begin{array}{l}0,3426 * * * \\
(3,69)\end{array}$ & & $\begin{array}{l}-0,5704 * * \\
(-2,36)\end{array}$ & $\begin{array}{l}0,3724 * * * \\
(2,79)\end{array}$ & $\begin{array}{l}0,3055^{* * * *} \\
(3,12)\end{array}$ & & & $\begin{array}{l}0,3273 \text { *** } \\
(3,14)\end{array}$ \\
\hline $\operatorname{AR}(3)$ & $\begin{array}{l}0,2285 \\
(1,98)\end{array}$ & & $\begin{array}{l}0,2419 \\
(1,02)\end{array}$ & & & & & \\
\hline $\mathrm{AR}(4)$ & $\begin{array}{l}-0,2163 \\
(-2,00)\end{array}$ & & $\begin{array}{l}-0,1293 \\
(-0,65)\end{array}$ & & & & & \\
\hline MA(1) & & $\begin{array}{l}0,0096 \\
(0,07)\end{array}$ & $\begin{array}{l}0,6289 * * * \\
(2,81)\end{array}$ & & & & & \\
\hline MA(2) & & $\begin{array}{l}0,4205 * * * \\
(3,97)\end{array}$ & $\begin{array}{l}1,0886 * * * \\
(5,75)\end{array}$ & & & & & \\
\hline MA(3) & & $\begin{array}{l}0,3443^{* *} \\
(2,33)\end{array}$ & $\begin{array}{l}0,2901 \\
(1,51)\end{array}$ & $\begin{array}{l}0,5052 * * * \\
(6,24)\end{array}$ & & $\begin{array}{l}0,3593^{* *} \\
(2,55)\end{array}$ & & $\begin{array}{l}0,3377 * * \\
(2,40)\end{array}$ \\
\hline MA(4) & & $\begin{array}{l}-0,0671 \\
(-0,48)\end{array}$ & $\begin{array}{l}0,6383 * * * \\
(3,91)\end{array}$ & $\begin{array}{l}-0,4013 * * * \\
(-4,36)\end{array}$ & & & $\begin{array}{l}-0,0981 \\
(-0,94)\end{array}$ & \\
\hline$\overline{R^{2}}$ & 0,18 & 0,24 & 0,28 & 0,27 & 0,09 & 0,08 & 0,01 & 0,17 \\
\hline Valor ajustado $R^{2}$ & 0,12 & 0,19 & 0,17 & 0,23 & 0,08 & 0,07 & $-0,01$ & 0,14 \\
\hline $\begin{array}{l}\text { Probabilidad chi-cuadrado } \\
\text { (Breusch-Godfrey) }\end{array}$ & 0,23 & 0,86 & 0,72 & 0,69 & 0,46 & 0,03 & 0,01 & 0,20 \\
\hline Error estándar de la regresión & 0,01 & 0,01 & 0,01 & 0,01 & 0,01 & 0,01 & 0,01 & 0,01 \\
\hline $\begin{array}{l}\text { Criterio de información de } \\
\text { Akaike }\end{array}$ & $-5,99$ & $-6,10$ & $-6,00$ & $-6,16$ & $-6,00$ & $-6,00$ & $-5,92$ & $-6,06$ \\
\hline $\begin{array}{l}\text { Criterio de información de } \\
\text { Schwarz }\end{array}$ & $-5,82$ & $-5,94$ & $-5,70$ & $-6,03$ & $-5,98$ & $-5,93$ & $-5,86$ & $-5,96$ \\
\hline Estadístico f (valor $p$ ) & 0,02 & 0,00 & 0,01 & 0,00 & 0,01 & 0,02 & 0,50 & 0,00 \\
\hline $\mathrm{N}^{\mathrm{o}}$ de observaciones & 65 & 69 & 65 & 67 & 67 & 69 & 69 & 67 \\
\hline
\end{tabular}

Fuente: Departamento Administrativo Nacional de Estadísticas (DANE) y cálculos del autor.

Notas:

Estadístico t entre paréntesis.

$* p<0,10$

$* * p<0,05$

$* * * p<0,01$

Estimación por mínimos cuadrados y métodos numéricos.

Errores estándar consistentes. 
CUADRO A6

Correlograma de residuos de la regresión seleccionada ${ }^{a}$

\begin{tabular}{|c|c|c|c|c|}
\hline Rezago & Autocorrelación & Correlación parcial & Estadístico Q & Probabilidad de estadístico Q \\
\hline 1 & 0,03 & 0,03 & 0,08 & \\
\hline 2 & 0,07 & 0,07 & 0,47 & \\
\hline 3 & $-0,03$ & $-0,03$ & 0,53 & 0,47 \\
\hline 4 & $-0,22$ & $-0,23$ & 4,13 & 0,13 \\
\hline 5 & $-0,16$ & $-0,15$ & 5,98 & 0,11 \\
\hline 6 & 0,01 & 0,05 & 5,99 & 0,20 \\
\hline 8 & $-0,04$ & $-0,11$ & 6,75 & 0,34 \\
\hline 9 & 0,22 & 0,15 & 10,59 & 0,16 \\
\hline 10 & $-0,01$ & 0,00 & 10,60 & 0,23 \\
\hline 11 & $-0,09$ & $-0,09$ & 11,33 & 0,25 \\
\hline 12 & 0,04 & 0,04 & 11,43 & 0,33 \\
\hline 13 & 0,00 & 0,08 & 11,44 & 0,41 \\
\hline 14 & $-0,10$ & $-0,08$ & 12,21 & 0,43 \\
\hline 17 & 0,09 & 0,16 & 13,25 & 0,58 \\
\hline 18 & $-0,13$ & $-0,22$ & 14,88 & 0,53 \\
\hline 19 & 0,08 & 0,06 & 15,46 & 0,56 \\
\hline 20 & $-0,02$ & 0,07 & 15,49 & 0,63 \\
\hline 21 & $-0,05$ & $-0,05$ & 15,78 & 0,67 \\
\hline 22 & 0,07 & $-0,03$ & 16,23 & 0,70 \\
\hline 23 & $-0,10$ & $-0,06$ & 17,28 & 0,69 \\
\hline 24 & 0,01 & 0,01 & 17,30 & 0,75 \\
\hline 25 & $-0,02$ & 0,01 & 17,33 & 0,79 \\
\hline 26 & 0,06 & $-0,02$ & 17,70 & 0,82 \\
\hline 27 & $-0,06$ & 0,00 & 18,16 & 0,84 \\
\hline 28 & 0,01 & $-0,05$ & 18,16 & 0,87 \\
\hline
\end{tabular}

Fuente: Departamento Administrativo Nacional de Estadísticas (DANE) y cálculos del autor.

a Véase la columna (1) del cuadro 2. 


\section{Bibliografía}

Agénor, P.R y P.J. Montiel (2008), Development Macroeconomics, Princeton, Princeton University Press.

Agénor, P.R., C.J. McDermott y E.S. Prasad (2000), "Macroeconomic fluctuations in developing countries: some stylized facts", World Bank Economic Review, vol. 14, N² ${ }^{\circ}$, Washington, D.C., Banco Mundial.

Ahmed, S. (2003), "Sources of economic fluctuations in Latin America and implications for choice of exchange rate regimes", Journal of Development Economics, vol. 72, $\mathrm{N}^{\circ} 1$, Amsterdam, Elsevier.

Barro, R.J. (1979), "Money and output in Mexico, Colombia, and Brazil", NBER Chapters, Cambridge, Massachusetts, National Bureau of Economic Research.

Blanchard, O.J. y D. Quah (1989), "The dynamic effects of aggregate demand and supply disturbances", American Economic Review, vol. 79, $\mathrm{N}^{\circ}$ 4, Nashville, Tennessee, American Economic Association.

Broda, C. (2004), "Terms of trade and exchange rate regimes in developing countries", Journal of International Economics, vol. $63, \mathrm{~N}^{\circ} 1$, Amsterdam, Elsevier.

Buiter, W.H. (1988), "Structural and stabilization aspects of fiscal and financial policy in the dependent economy", Oxford Economic Papers, New Series, vol. 40, № 2, Oxford, Oxford University Press.

Campbell, J.Y. y G. Mankiw (1987), "Are output fluctuations transitory?", The Quarterly Journal of Economics, vol. 102, $\mathrm{N}^{\circ}$ 4, Oxford, Oxford University Press.

Edwards, S. (1986), "Are devaluations contractionary?", The Review of Economics and Statistics, vol. 68, $\mathrm{N}^{\circ} 3$, Massachusetts, The MIT Press.

(1983), "The short-run relation between growth and inflation in Latin America: comment", American Economic Review, vol. 73, $\mathrm{N}^{\circ} 3$, Nashville, Tennessee, American Economic Association.

Frankel, J.A. (2010), "The natural resource curse: a survey", NBER Working Paper, $\mathrm{N}^{\circ} 15836$, Cambridge, Massachusetts, National Bureau of Economic Research.

Greenwood, J. (1984), "Non-traded goods, the trade balance, and the balance of payments", The Canadian Journal of Economics, vol. $17, \mathrm{~N}^{\circ} 4$, Wiley.

Hoffmaister, A.W. y J.E. Roldós (2001), "The sources of macroeconomic fluctuations in developing countries: Brazil and Korea”, Journal of Macroeconomics, vol. 23, $\mathrm{N}^{\circ}$ 2, Amsterdam, Elsevier.

Hoffmaister, A.W., J.E. Roldós y P. Wickham (1998), "Macroeconomic fluctuations in sub-Saharan Africa", IMF Staff Papers, vol. 45, $\mathrm{N}^{\circ}$ 1, Houndmills, Palgrave Macmillan.

Holland, A. y A. Scott (1998), "The determinants of UK business cycles", Economic Journal, vol. 108, № 449, Wiley.

Izquierdo, A., R. Romero y E. Talvi (2007), "Business cycles in Latin America: the role of external factors", Documento de Trabajo, Washington, D.C., Banco Interamericano de Desarrollo.

Kaminsky, G.L. (2010), "Terms of trade shocks and fiscal cycles", NBER Working Paper Series, $\mathrm{N}^{\circ} 15780$, Cambridge, Massachusetts, National Bureau of Economic Research.

Kent, C.J. y P. Cashin (2003), "The response of the current account to terms of trade shocks: persistence matters", IMF Working Papers, $\mathrm{N}^{\circ}$ 03/143, Washington, D.C., Fondo Monetario Internacional.

Kose, M.A. (2002), "Explaining business cycles in small open economies: how much do world prices matter?", Journal of International Economics, vol. 56, $\mathrm{N}^{\circ}$ 2, Amsterdam, Elsevier.

Kose, M.A. y R.G. Riezman (1999), "Trade shocks and macroeconomic fluctuations in Africa", CESifo Working Paper Series, $\mathrm{N}^{\circ} 203$, Munich, Center for Economic Studies and Ifo Institute for Economic Research.
Krugman, P. y L. Taylor (1978), "Contractionary effects of devaluation", Journal of International Economics, vol. 8, $\mathrm{N}^{\circ} 3$, Amsterdam, Elsevier.

Lizondo, J.S. y P.J. Montiel (1989), "Contractionary devaluation in developing countries: an analytical overview", IMF Staff Papers, vol. 36, $\mathrm{N}^{\circ} 1$, Houndmills, Palgrave Macmillan.

MacKinnon, J.G., A.A. Haug y L. Michelis (1999), "Numerical distribution functions of likelihood ratio tests for cointegration", Journal of Applied Econometrics, vol. 14, $\mathrm{N}^{\circ}$ 5, John Wiley \& Sons.

Mahadeva, L. y J. Gómez (2009), "The international cycle and Colombian monetary policy", Borradores de Economía, $\mathrm{N}^{\circ} 557$, Bogotá, Banco de la República.

Mankiw, N.G. (1989), "Real business cycles: a new Keynesian perspective", The Journal of Economic Perspectives, vol. 3, $\mathrm{N}^{\circ}$ 3, Nashville, Tennessee, American Economic Association.

Medina, L. (2010), "The dynamic effects of commodity prices on fiscal performance in Latin America", IMF Working Papers, vol. 192, $\mathrm{N}^{\circ}$ 201, Washington, D.C., Fondo Monetario Internacional.

Mendoza, E.G. (1995), "The terms of trade, the real exchange rate, and economic fluctuations", International Economic Review, vol. 36, $\mathrm{N}^{\circ}$ 1, Pennsylvania, Universidad de Pennsylvania/ Osaka University Institute of Social and Economic Research Association.

Montenegro, A. (2002), Series de tiempo, Bogotá, Pontificia Universidad Javeriana.

Nelson, C.R. y C.L. Plosser (1982), "Trends and random walks in macroeconomic time series: some evidence and implications", Journal of Monetary Economics, vol. 10, $\mathrm{N}^{\circ}$ 2, Amsterdam, Elsevier.

Obstfeld, M. (1982), "Aggregate spending and the terms of trade: is there a Laursen-Metzler effect?", The Quarterly Journal of Economics, vol. 97, $\mathrm{N}^{\circ}$ 2, Oxford, Oxford University Press.

Parra, J.C. (2008), "Hechos estilizados de la economía colombiana: Fundamentos empíricos para la construcción y evaluación de un modelo DSGE", Borradores de Economía, N 4602, Bogotá, Banco de la República.

Raddatz, C. (2007), "Are external shocks responsible for the instability of output in low-income countries?", Journal of Development Economics, vol. 84, $\mathrm{N}^{\circ} 1$, Amsterdam, Elsevier.

Rand, J. y F. Tarp (2002), "Business cycles in developing countries: are they different?", World Development, vol. $30, \mathrm{~N}^{\circ} 12$, Amsterdam, Elsevier.

Razmi, A. (2007), "The contractionary short-run effects of nominal devaluation in developing countries: some neglected nuances", International Review of Applied Economics, vol. 21, $\mathrm{N}^{\circ} 5$, Abingdon, Routledge.

Reinhart, C.M. y V.R. Reinhart (1991), "Output fluctuations and monetary shocks: evidence from Colombia", IMF Staff Papers, vol. 38, N ${ }^{\circ}$, Houndmills, Palgrave Macmillan.

Shapiro, M.D. y M.W. Watson (1989), "Sources of business cycle fluctuations", NBER Working Papers, $\mathrm{N}^{\circ} 2589$, Cambridge, Massachusetts, National Bureau of Economic Research.

Svensson, L.E.O. y A. Razin (1983), "The terms of trade and the current account: the Harberger-Laursen-Metzler effect", The Journal of Political Economy, vol. 91, $\mathrm{N}^{\circ}$ 1, Chicago, The University of Chicago Press.

Tornell, A. y P.R. Lane (1999), "The voracity effect", American Economic Review, vol. 89, $\mathrm{N}^{\circ} 1$, Nashville, Tennessee, American Economic Association. 